\title{
Analysis of Discharge Channel Characteristics Based on Three-Phase Flow Dielectric Characteristics in USV-MF Assisted WEDM-LS
}

Yan Wang ( $\sim$ yanwang909909@163.com)

University of Shanghai for Science and Technology

Jie Yang

University of Shanghai for Science and Technology

Wei Xiong

University of Shanghai for Science and Technology

Hua-Yi Chai

University of Shanghai for Science and Technology

Rui Wang

University of Shanghai for Science and Technology

\section{Original Research}

Keywords: three-phase flow, USV-MF assisted, WEDM-LS, discharge channel, workpiece with large thickness

Posted Date: February 17th, 2021

DOI: https://doi.org/10.21203/rs.3.rs-201909/v1

License: (c) (1) This work is licensed under a Creative Commons Attribution 4.0 International License. Read Full License

Version of Record: A version of this preprint was published at The International Journal of Advanced Manufacturing Technology on August 13th, 2021. See the published version at https://doi.org/10.1007/s00170-021-07841-6. 


\title{
Analysis of discharge channel characteristics based on three-phase
}

\section{flow dielectric characteristics in USV-MF assisted WEDM-LS}

\author{
Yan Wang ${ }^{\mathrm{a}, *}$, Jie Yang ${ }^{\mathrm{a}}$, Wei Xiong ${ }^{\mathrm{b}}$, Hua-Yi Chai ${ }^{\mathrm{a}}$, Rui Wang ${ }^{\mathrm{a}}$ \\ ${ }^{a}$ School of Mechanical Engineering, University of Shanghai for Science and \\ Technology, Shanghai 200093, China \\ ${ }^{b}$ School of Materials Science and Engineering, University of Shanghai for Science \\ and Technology, Shanghai 200093, China \\ ${ }^{*}$ Corresponding authors: \\ E-mail address: yanwang909909@163.com (Y. Wang)
}

\begin{abstract}
In this paper, considering the effects of the characteristics of the three-phase flow dielectric in the discharge gap flow field on the discharge channels in the ultrasonic vibration and magnetic field assisted low speed WEDM, the model of discharge channel including discharge position and size of discharge channel is established and analyzed. Firstly, given working conditions of cutting workpiece with large thickness, a mechanism of bubble-particle bridging breakdown was proposed. Then a prediction model of the discharge point position in USV-MF assisted WEDM-LS under three-phase flow dielectric characteristics based on the breakdown mechanism was performed, and FEM software COMSOL was used to simulate and analyze this model. By comparing with the experimental results of discharge point position captured by high speed camera, the accuracy of the prediction results of discharge point position in USV-MF assisted WEDM-LS was verified, showing that this model can more accurately explain the dielectric breakdown in cutting workpiece with large thickness. Next, the model of radius of discharge channel including magnetic field intensity, electric field intensity, medium pressure and surface tension of discharge channel was established, and the influence of ultrasound and magnetic field on the size of discharge channel was analyzed theoretically. The radius of discharge channel in USV-MF assisted WEDM-LS was obtained by solving the partial differential equation in MATLAB. According to discharge point position and radius of the plasma discharge channel, surface morphology of workpiece was simulated by COMSOL. Finally, the experiment of photographing the discharge channel in USV-MF assisted WEDMLS was carried out to verify the above model. It shows that the trend of the simulated results of discharge channel position is the same as that of the experimental results, and the error between the peak height value of the simulated surface morphology of workpiece and that of the experimental surface morphology of workpiece is within $6 \%$.
\end{abstract}

Keywords: three-phase flow; USV-MF assisted; WEDM-LS; discharge channel; workpiece with large thickness

\section{Introduction}

WEDM is a non-traditional machining technology, in which a continuously moving electrode wire is used as tool electrode to produce spark discharge heat between electrode wire and workpiece to melt materials. It plays an important role in the field of precision manufacturing, as reported by Abbas et al. [1] However, traditional WEDM 
can no longer meet the increasingly high requirements for machining. It is rather difficult to cut workpiece with large thickness and the machining efficiency is low. Therefore, a processing method of USV-MF assisted WEDM-LS is presented in this paper to improve the processing performance.

In the aspect of complex processing technology, an ultrasonic vibration assisted EDM technology was developed and compared with traditional EDM. The results showed that the $R a$ value was reduced by $14 \%$ in the center of the workpiece machined area and $20 \%$ in the borders [2]. Chen et al. [3] studied the motion trajectory of electron beam and debris in magnetic field assisted WEDM. And it indicated that additional (extra) constant magnetic field can increase the discharge crater volume and the expel efficiency of charged debris, which improved the machining quality. Wang et al. [4] found that the machining efficiency and the surface quality of workpiece are significantly increased by USV-MF assisted WEDM-LS. In the aspect of discharge channel, Dhanik et al. [5] developed a mathematical model of discharge channel in the deionized water during micro-EDM. In addition, the distributions of temperature and pressure in the discharge channel as well as the time-varying radius of discharge channel were calculated under given conditions. Chu et al. [6] investigated the law of discharge channel expansion in micro-EDM considering the effect of magnetic field generated by the discharge current, and a time-varying plasma channel expansion model of micro-EDM was established. However, many scholars tend to describe the effects of bubbles and debris on discharge conditions in discharge gap during discharge process macroscopically without explaining the reason and mechanism for this phenomenon microscopically. Besides, there is no related research in theoretical calculations of the radius of discharge channel in USV-MF assisted WEDM-LS.

In this paper, starting from the three-phase flow dielectric in the discharge gap, the influence of the three-phase flow dielectric on the distortion of the electric and magnetic fields between two electrodes is obtained through theoretical calculation and simulation analysis, and then the influence of the three-phase flow dielectric on the discharge channel was analyzed. Firstly, a mechanism of bubble-particle bridging breakdown suitable for WEDM-LS machining workpiece with large thickness is proposed. Based on the breakdown mechanism, a prediction model of the discharge channel under that condition was established. Then the discharge channel location was obtained by COMSOL and MATLAB. The time-varying radius model of discharge channel in USVMF assisted WEDM-LS was built and the influence of ultrasonic vibration and magnetic field on the radius and size of discharge channel was analyzed. The mechanism of USV-MF assisted WEDM-LS was explained from the view of discharge channel and micro level. Finally, an experiment of photographing the discharge channel in the USV-MF assisted WEDM-LS of workpiece with large thickness was accomplished, and the results show that the discharge channel characteristics in USVMF assisted WEDM-LS of can be predicted well by the above model. 


\section{Principle of USV-MF assisted WEDM-LS}

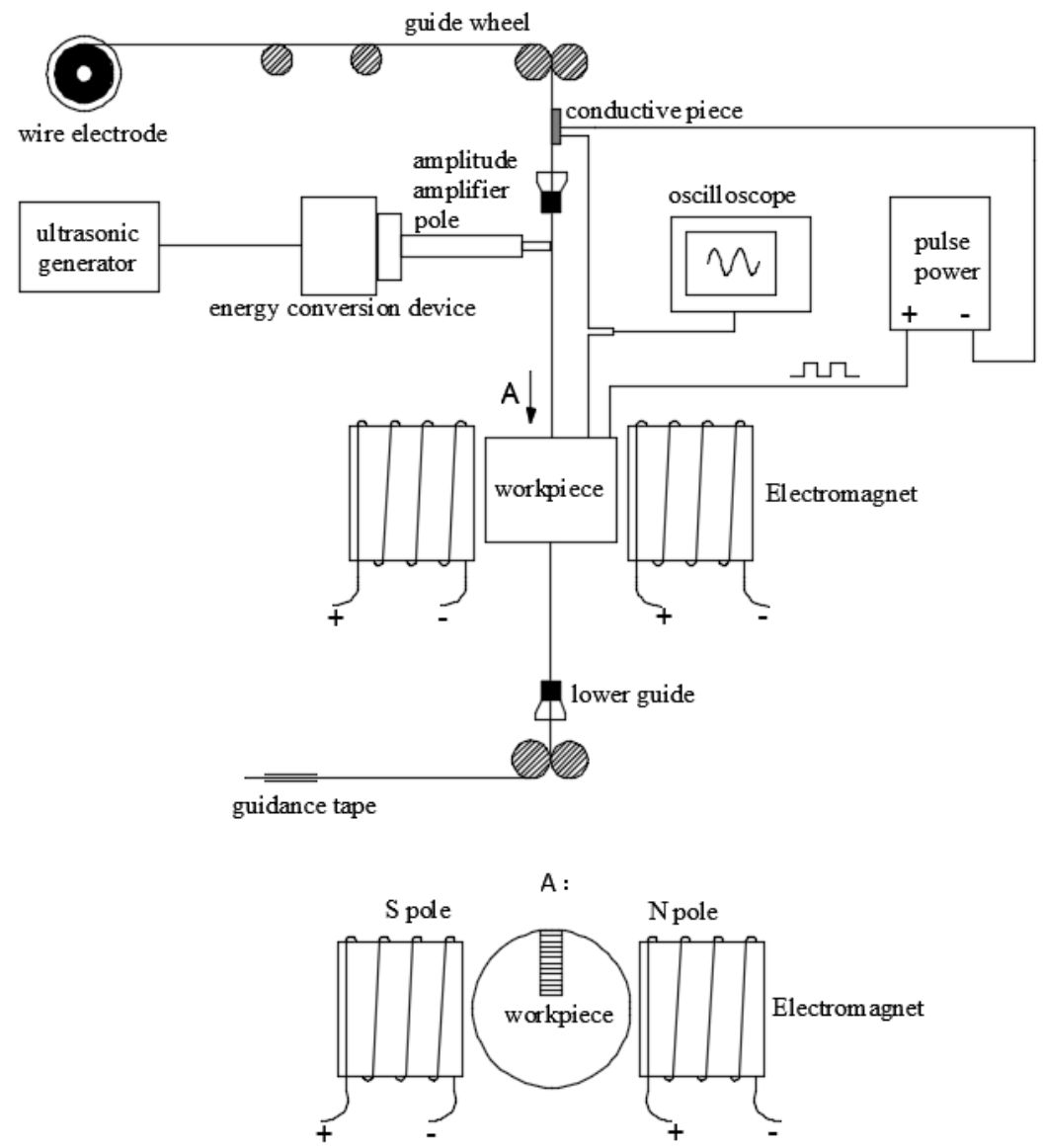

Fig. 1 Schematic of USV-MF assisted WEDM-LS system

The schematic of USV-MF assisted WEDM-LS is shown in Fig.1. The ultrasonic device consists of an ultrasonic generator, an energy conversion device, and an amplitude amplifier pole. When the ultrasonic device is activated, high-frequency mechanical vibration is generated by the transducer and amplified into a certain amplitude by the pole. The end of the pole is in contact with the electrode wire, transmitting high-frequency vibration to the electrode wire, which makes the electrode wire vibrate at high frequency. On the one hand, the vibration of the electrode wire is changed. On the other hand, the high-frequency vibration of the electrode wire causes severe disturbance to the discharge gap flow field. The bubbles generated by the discharge in the discharge gap are broken because of the disturbance of the electrode wire, which reduces the phenomenon of abnormal discharge during the WEDM-LS. The magnetic field device consists of two cylindrical electromagnets with parallel ends to ensure that a uniform magnetic field is generated in the processing area. The magnetic field strength can be changed by changing the current flowing through the coil. During the WEDM-LS, the charged particles in the discharge channel are affected by the magnetic field, which leads to the change of the shape and position of discharge channel.

\section{Discharge model based on a bubble-particle bridging breakdown mechanism}

Because workpiece with large thickness in WEDM-LS is considered in this paper, 
the debris removal condition will be greatly affected by the thickness of workpiece, which causes some erosion products enter the discharge channel with bubbles generated by discharge. The air bubbles and the erosion products in the dielectric undergo ionization under the action of the electric field, and the ionized positive and negative charges are arranged in the discharge channel along the direction of the electric field line, which is equivalent to reducing the electrode gap. In addition, the presence of tiny debris will also cause distortion of the inter-electrode electric field, and dielectric breakdown is most likely to occur at the place with the local maximum field strength, forming a discharge channel.

Therefore, the single bubble mechanism is not suitable for the dielectric breakdown phenomenon in the WEDM-LS process, a mechanism of bubble-particle bridging breakdown is proposed in this paper. Under this mechanism, the breakdown of the dielectric in the initial state of the discharge is dominated by the bubble breakdown mechanism. As the discharge machining proceeds, the concentration of bubbles and erosion products generated by the discharge increases, and the bubble breakdown mechanism is no longer applicable. The dielectric breakdown under this working condition can explain more accurately by the mechanism of bubble-particle bridging breakdown.

\subsection{Electric field strength under bubble breakdown mechanism}

During the WEDM-LS process of workpiece with large thickness, a pulse voltage is applied between the electrode wire and the workpiece, and the inter-electrode field strength is continuously enhanced with the feed of the electrode wire. As the inter electrode distance decreases, the abrupt changes in the macro curvature of the contour of the workpiece and the micro unevenness on the surface of the electrode wire will increase the non-uniformity of the electric field intensity distribution between the poles. Based on the field-electron emission theory, some free electrons will pass through the potential barrier on the electrode surface forming electron emission under the effect of the tunneling effect, and then a pre-discharge current before breakdown occurs as shown in Fig.2.

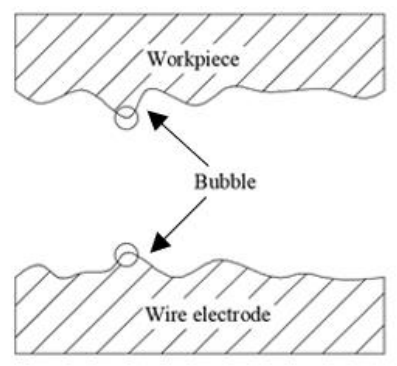

(a) Pre-breakdown state

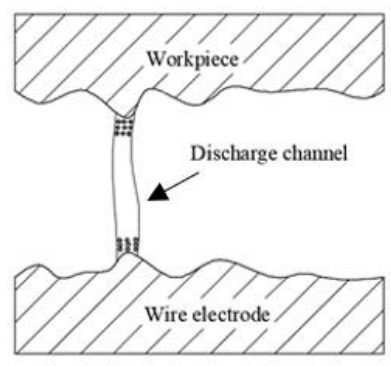

(b) Initial state

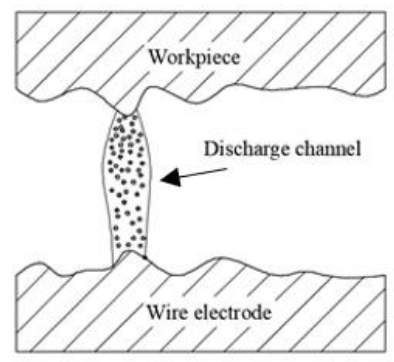

(c) Balance state

Fig. 2 The formation process of the discharge channel under the bubble breakdown mechanism

The current density due to cathode electron emission can be expressed by the Fowler and Nordheim equation [7].

$$
j=C E_{P}^{2} \exp \left(-\frac{D}{E_{P}}\right)
$$


Where, $j$ is the current density, $E_{P}$ is an enhanced electric field, $C$ and $D$ is a constant.

It can be known from Eq. (1) that the current density is related to electric field strength and the higher the electric field strength, the higher the current density. Considering the geometry of the micro peaks on the electrode surface, the electric field strength at the micro peaks can be expressed as:

$$
E_{\mathrm{p}}=\frac{U}{r_{\mathrm{t}}^{0.75} l_{\mathrm{g}}^{0.25}}
$$

Where, $r_{\mathrm{t}}$ is the tip radius of the micro peak, $U$ is the inter-electrode voltage, and $l_{\mathrm{g}}$ is the discharge gap. Since $l_{\mathrm{g}}>r_{t}, E_{P}$ will be significantly larger than the average electric field strength $E_{a}=\frac{U}{l_{\mathrm{g}}}$. Therefore, the breakdown of inter polar dielectric will first appear at the micro peak. According to Eq. (2), the electric field strength at the micro peak of the nearest point between the electrode wire and the workpiece under the bubble breakdown mechanism can be obtained. When $E_{P}>E_{0}$ (the electric field strength when the liquid dielectric breakdown occurs), bubbles are generated at the micro peaks and the discharge breakdown process begins.

\subsection{Electric field strength under the particle bridging breakdown mechanism}

In the WEDM-LS, the metal particles eroded by the discharge and the hightemperature air bubbles generated during the discharge erosion cannot be removed from the discharge gap in a short time. These metal particles and air bubbles will cause a distortion effect on the electric field in the discharge gap and affect the liquid insulativity in the discharge gap, leading to the change of discharge point position eventually. Fig. 3 shows the process of the discharge channel formation under the particle bridging breakdown mechanism.

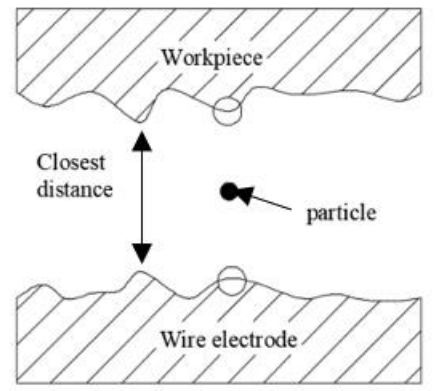

(a) Pre-breakdown state

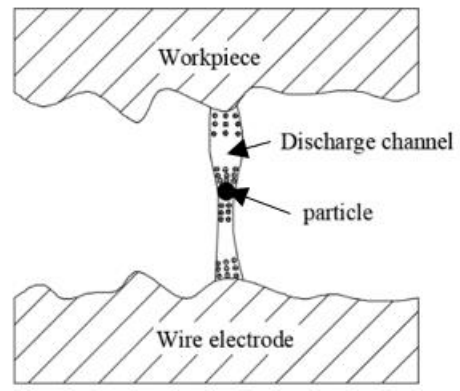

(b) Initial state

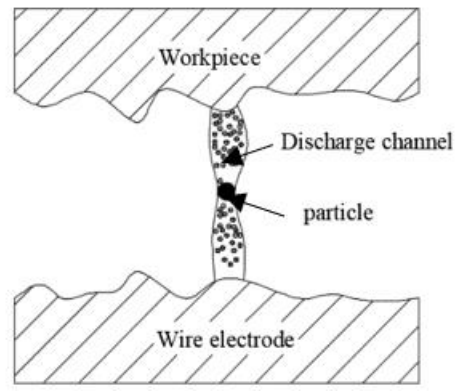

(c) Balance state

Fig. 3 The formation process of the discharge channel of the "particle" bridge breakdown mechanism

From Fig. 3, it can be known that due to the existence of particles in the discharge gap, the formation position of the discharge channel is no longer the nearest point 
between the electrode wire and the workpiece. The particle bridging causes the dielectric breakdown, and then the discharge channel is generated. This is because in a narrow discharge gap, the particles cause an increase in the electric field strength, and the presence of the particles reduces the dielectric insulation. According to the principle of dielectric breakdown, it can be concluded that the possibility of discharge breakdown is most likely to occur there. In order to better explain the above situation, a model of discharge gap electric field distortion is established in this paper. Fig. 4 is a schematic of the electric field distortion in the discharge gap.

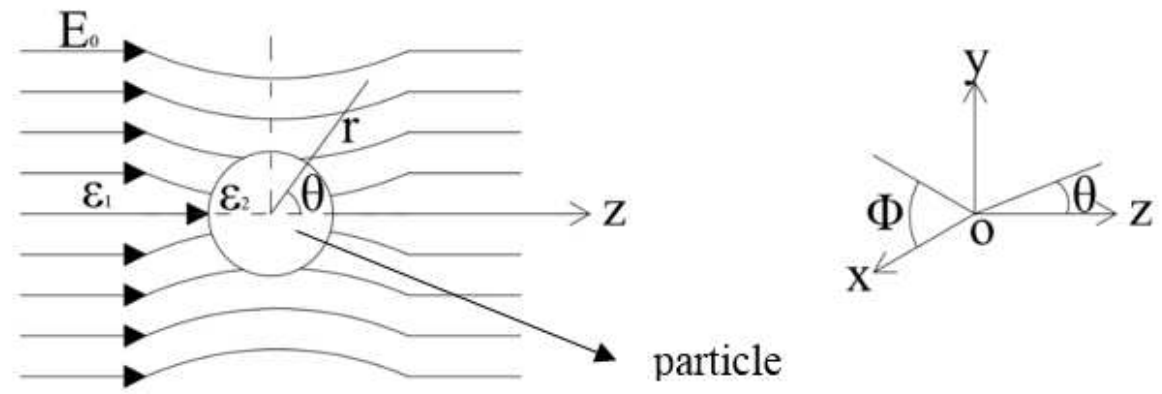

Fig. 4 Schematic of electric field distortion

As shown in Fig. 4, solving the Lagrangian equation in the spherical coordinate system, the distribution of electric field inside and outside the bubble under a uniform electric field can be obtained. Let the radius of the bubble be $a$, the voltage inside and outside the ball are $V_{1}$ and $V_{2}$, and the dielectric constant of the liquid dielectric is $\varepsilon_{1}$, the dielectric constant of the particle is $\varepsilon_{2}$. Due to the symmetry of the problem, the voltage $V$ has nothing to do with the azimuth angle $\varphi$ and is only a function of $\mathrm{r}$ and $\theta$.

The general solution of Laplace equation for axisymmetric problems in spherical coordinates is shown in Eq. (3) [8]:

$$
\mathrm{V}(\mathrm{r}, \theta)=\sum_{n}\left(A_{n} r^{n}+\frac{B_{n}}{r^{n+1}}\right) P_{n}(\cos \theta)
$$

Where, $P_{n}(\cos \theta)$ is the Legendre function, $A_{n}$ and $B_{n}$ are the coefficients.

The definite solution conditions of this problem are:

(1) The electric field is still uniform far away from the bubble:

$$
V_{1}=-E_{0} r \cos \theta
$$

(2) The potential on the sphere is continuous:

$$
V_{1}=V_{2}
$$

(3) There is no free charge on the surface of the bubble, and the normal component of the electric field strength on the sphere is equal:

$$
\varepsilon_{1} \frac{\partial V_{1}}{\partial r}=\varepsilon_{2} \frac{\partial V_{2}}{\partial r}
$$

(4) When $r=0, V \neq \infty$.

The electric field strength in the $r$ direction outside the bubble can be obtained:

$$
E_{r 1}=\frac{\partial V_{1}}{\partial r}=\left[1+\frac{2 a^{3}}{r^{3}}\left(\frac{\varepsilon_{2}-\varepsilon_{1}}{\varepsilon_{2}+2 \varepsilon_{1}}\right)\right] E_{0} \cos \theta
$$

The electric field strength in the $\theta$ direction outside the bubble can be obtained:

$$
E_{\theta 1}=\frac{\partial V_{1}}{r \partial \theta}=\left[1-\frac{a^{3}}{r^{3}}\left(\frac{\varepsilon_{2}-\varepsilon_{1}}{\varepsilon_{2}+2 \varepsilon_{1}}\right)\right] E_{0} \cos \theta
$$


So the electric field strength at the position of particles can be calculated as shown in Eq.9:

$$
E_{z 2}=-\frac{\partial V_{2}}{\partial z}=E_{0} \frac{3 \varepsilon_{1}}{\varepsilon_{2}+2 \varepsilon_{1}}
$$

In general, $\varepsilon_{1}>\varepsilon_{2}$. According to the above formula, the electric field strength in the bubble is uniform and enhanced. The enhancement factor of electric field strength is related to the dielectric constants of the liquid dielectric and the bubble, and has nothing to do with the electric field strength outside the bubble.

\subsection{Electrode wire vibration model in USV-MF assisted WEDM-LS}

In this paper, the magnetic induction line of the applied magnetic field is perpendicular to the electrode wire. During the machining process, the current passes through the electrode wire, and it can be known that the electrode wire is subjected to the Ampere force according to Ampere's law, which causes the vibration response of the electrode wire to change. Vibration response of the electrode wire during a single discharge under the action of magnetic field $U_{e}(x, t)$ can be expressed as [9]:

$$
\begin{aligned}
& U_{e}^{\prime}(x, t)=\frac{2}{L} \sum_{i=1}^{\infty} \frac{\sin \left(\frac{\omega_{i}}{c} x\right)}{\rho_{l} \omega_{i}^{2} A} \sin \left(\frac{\omega_{i}}{c} \lambda\right) F_{e}{ }^{\prime}\left[n e^{n t} \sin A t+A\left(1-e^{n t} \cos A t\right)\right] \\
& +e^{n t} \sin \left(\frac{\omega_{i}}{c} x\right)\left[\cos A t-\frac{n \sin A t}{A^{2}}\right] \int_{0}^{L} U_{0}(\eta) \sin \left(\frac{\omega_{i}}{c} \eta\right) d \eta+\left[\frac{e^{n t} \sin \left(\frac{\omega_{i}}{a} x\right) \sin A t}{A^{2}}\right] \int_{0}^{L} V_{0}(\eta) \sin \left(\frac{\omega_{i}}{c} \eta\right) d \eta
\end{aligned}
$$

where $a=\sqrt{\mathrm{T}_{\mathrm{s}} / \rho_{l}}, T_{s}$ is the tension of the electrode wire, $E$ is the elastic modulus, $\rho_{l}$ is the linear density of the electrode wire, $\lambda$ is the discharge position on the wire electrode, $\omega_{i}$ is the natural frequency of the wire, $i$ is the frequency of order, $\boldsymbol{F}_{\boldsymbol{e}}$ is the resultant force which consists of discharging force, electrostatic force and Ampere force, $n$ is the damping factor and equals to $-\psi \omega_{i} \psi, \eta$ is the span of the electrode wire, $U_{0}$ and $V_{0}$ are the initial displacement and the initial speed of the electrode wire, respectively.

The vibration response $U_{u}(x, t)$ of the electrode wire under ultrasonic excitation can be expressed as

$$
\left\{\begin{array}{l}
U_{u}(x, t)=\frac{\mathbf{F}_{0}}{l \rho_{l}} \sum_{i=1}^{\infty} \frac{\sin \left(\frac{\omega_{i}}{a}\right) \sin \left(\frac{\omega_{i}}{a} \eta\right)}{\rho_{l}}\left\{A_{n}+C_{n}+\left(B_{n}+D_{n}\right)\left[\cos \omega t-e^{n t} \cos \left(\sqrt{\omega_{i}^{2}-n^{2}}\right) t\right]\right\} \\
A_{n}=\frac{n\left(e^{n t} \sin \left(\sqrt{\omega^{2}-n^{2}}\right) t-\sin \omega t\right)}{\omega^{2}-2 \omega \sqrt{\omega_{i}{ }^{2}-n^{2}}+\omega_{i}{ }^{2}} \\
B_{n}=\frac{\sqrt{\omega_{i}{ }^{2}-n^{2}-\omega}}{\omega^{2}-2 \omega \sqrt{\omega_{i}{ }^{2}-n^{2}+\omega^{2}}} \\
C_{n}=\frac{n\left(e^{n t} \sin \left(\sqrt{\omega_{i}^{2}-n^{2}}\right) t+\sin \omega t\right)}{\omega^{2}+2 \omega \sqrt{\omega_{i}{ }^{2}-n^{2}}+\omega_{i}{ }^{2}} \\
D_{n}=\frac{\sqrt{\omega_{i}{ }^{2}-n^{2}}+\omega}{\omega^{2}-2 \omega \sqrt{\omega_{i}{ }^{2}-n^{2}}+\omega^{2}}
\end{array}\right.
$$

where $l$ is the span of the electrode wire and $\eta$ is the position where the ultrasonic excitation is applied on the electrode wire.

Since the electrode wire is a linear system, the vibration response of the electrode wire under the combined effect of ultrasound and magnetic field is obtained as the vector sum of Eq. (10) and Eq. (11). 


$$
\vec{U}=\overrightarrow{U_{e}^{\prime}}+\overrightarrow{U_{u}}
$$

Define $f(x, y)$ as the model of single-discharge etch pit, and $g(x, y)$ as the expression of the surface morphology of the workpiece. $a, b$ are the relative positions of the discharge points on the workpiece surface. Therefore, the surface morphology of the workpiece after each discharge can be expressed as:

$$
g(x, y)=g(x, y)-\sum_{i=1}^{n} f\left(\left(x-a_{i}\right),\left(y-b_{i}\right)\right)
$$

Define $l_{g}$ as the shortest distance between the electrode wire and the workpiece during processing, so $l_{g}$ can be expressed as:

$$
l_{g}=\min (|g(x, y)-U(x, t)|)
$$

\subsection{Bubble trajectory simulation}

\subsubsection{Governing Equation}

In this paper, the level set method is used to track the bubbles generated by the discharge. The level set function $\phi(r, z, t)$ is defined in the entire calculation domain to describe the interface between the bubbles and deionized water [10].

$$
\phi(r, z, t) \begin{cases}<0.5, & \text { Bubble; } \\ =0.5, & \text { Interface ; } \\ >0.5, & \text { Deionized water; }\end{cases}
$$

The governing equation of the level set function is as follows.

$$
\begin{gathered}
\frac{\partial \phi}{\partial t}+\mathbf{u} \cdot \nabla \phi=\gamma \nabla \cdot\left(\varepsilon \nabla \phi-\phi(1-\phi) \frac{\nabla \phi}{|\nabla \phi|}\right) \\
\rho\left(\frac{\partial \mathbf{u}}{\partial t}+\mathbf{u} \cdot \nabla \mathbf{u}\right)=-\nabla p+\nabla \cdot \mu \nabla \mathbf{u}+\nabla \mathbf{u}^{T}+\rho \mathbf{g}+\mathbf{F}_{\mathrm{st}} \\
\nabla \cdot \mathbf{u}=0
\end{gathered}
$$

Where, $\mathbf{u}$ is the fluid velocity, $\gamma$ and $\varepsilon$ are the re-initialization parameters, $\gamma$ determines the number of re-initialization, $\varepsilon$ is a parameter that determines the thickness of the interface, usually half of the maximum grid size, $\rho$ is the global density, $p$ is the global pressure, and $t$ is Time, $\rho \mathbf{g}$ is the gravity term in the momentum equation, and $\mathbf{F}_{\text {st }}$ is the surface tension component, which can be expressed by Eq. (19).

$$
\mathbf{F}_{\mathrm{st}}=\nabla \cdot\left[\begin{array}{lll}
\sigma & \mathbf{I}+-\mathbf{n n}^{T} & \delta
\end{array}\right]
$$

Where $\sigma$ is surface tension coefficient, $\mathbf{I}$ is unit matrix, $\mathbf{n}$ is interface unit normal vector which can be defined by Eq. (20), and $\delta$ is Dirac delta function defined by Eq. (21). 


$$
\begin{gathered}
\mathbf{n}=\frac{\nabla \phi}{|\nabla \phi|} \\
\delta=6|\phi(1-\phi) \| \nabla \phi|
\end{gathered}
$$

Because the level set function is continuous in the entire domain and is a smooth step function, it can also be used to determine the global density and dynamic viscosity with the following formula.

$$
\begin{aligned}
& \rho=\rho_{\mathrm{w}}+\rho_{\mathrm{a}}-\rho_{\mathrm{w}} \phi \\
& \rho=\rho_{\mathrm{w}}+\rho_{\mathrm{a}}-\rho_{\mathrm{w}} \phi
\end{aligned}
$$

Where, $\rho_{\mathrm{w}}$ and $\mu_{\mathrm{w}}$ represent the density and viscosity of water, $\rho_{a}$ and $\mu_{a}$ represent the density and viscosity of the gas in the bubble.

\subsubsection{Initial and boundary conditions}

In order to facilitate photographing the discharge channel of WEDM-LS, immersed machining method is used in this paper. The analysis shows that the model has a high degree of symmetry, so the three-dimensional space model can be converted into a twodimensional plane model for the sake of simplifying calculation. Fig. 5 shows the calculation area and boundary conditions of bubble tracking.

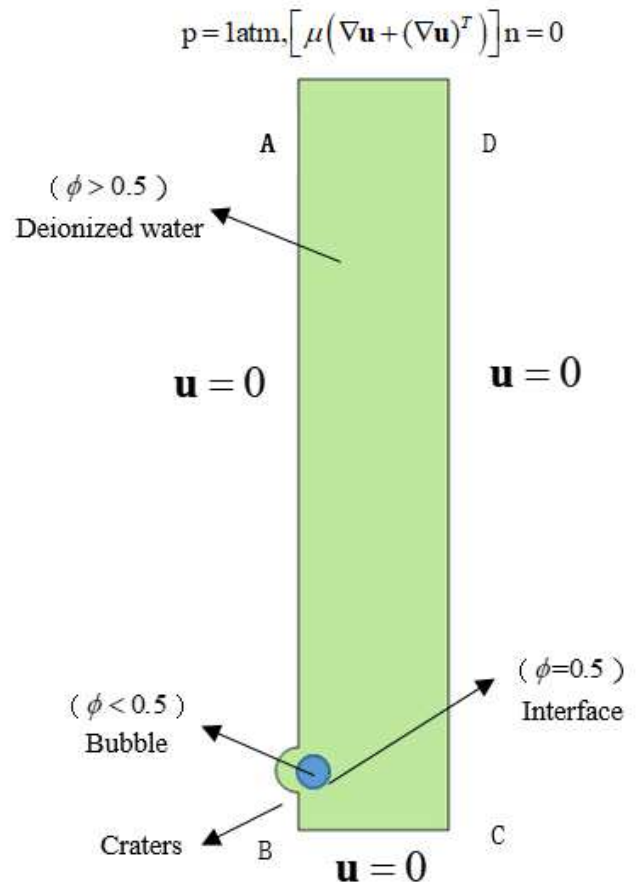

Fig. 5 Schematic diagram of bubble tracking calculation

As shown in Fig. 5, the $\mathrm{AB}$ boundary is the workpiece, the $\mathrm{CD}$ boundary is the electrode wire, $\mathrm{AD}$ is the open boundary, and $\mathrm{BC}$ is the closed boundary. The boundary equation of each boundary is shown in Fig. 5. The entire model calculation area is the discharge gap between the electrode wire and the workpiece. Where $\phi<0.5$ represents air bubbles, $\phi>0.5$ represents deionized water, $\phi=0.5$ represents the 
interface between air bubbles and deionized water. The following assumptions are made to simplify the model:

1. Only one bubble is generated per discharge;

2. The radius of the bubble does not change during the movement of bubble;

3. When the bubble breaks under the action of ultrasound, the bubble disappears. In the traditional WEDM-LS, although there is free vibration of the electrode wire at the CD boundary, the disturbance of the free vibration to the region can be ignored. So the boundary equation of the CD boundary in the traditional WEDM-LS is $\mathbf{u}=0$. When the ultrasonic vibration acts on the electrode wire, the electrode wire vibrates violently, which has a huge impact on the discharge gap flow field and cannot be ignored. The boundary conditions of the $\mathrm{CD}$ boundary under ultrasonic excitation can be set as:

$$
\mathbf{u}=\mathbf{A} \cos (2 \pi f t+\varphi)
$$

Where, $\mathbf{A}$ is the amplitude of the ultrasonic vibration, $f$ is the frequency of the ultrasonic vibration, $t$ is time, and $\varphi$ is the phase angle.

Because the bubble tracking model involves the Navier-Stokes equations in fluid mechanics, the complex partial differential equations need to be solved. The calculation is implemented in the Live MATLAB module of Comsol Multiphysics software, and the Projection method is used as solution algorithm [11]. It is divided into two steps: firstly, the intermediate velocity that does not meet the continuity condition is calculated without considering the pressure gradient, and then the accurate pressure field is obtained; secondly based on the obtained pressure field, the intermediate velocity is projected into the non-divergent velocity vector space to obtain an accurate speed field at next moment. Then the speed and pressure solved are iterated into the level set function, and finally the distribution of the level set function at this time step is obtained. The solution process is shown in Fig. 6. 


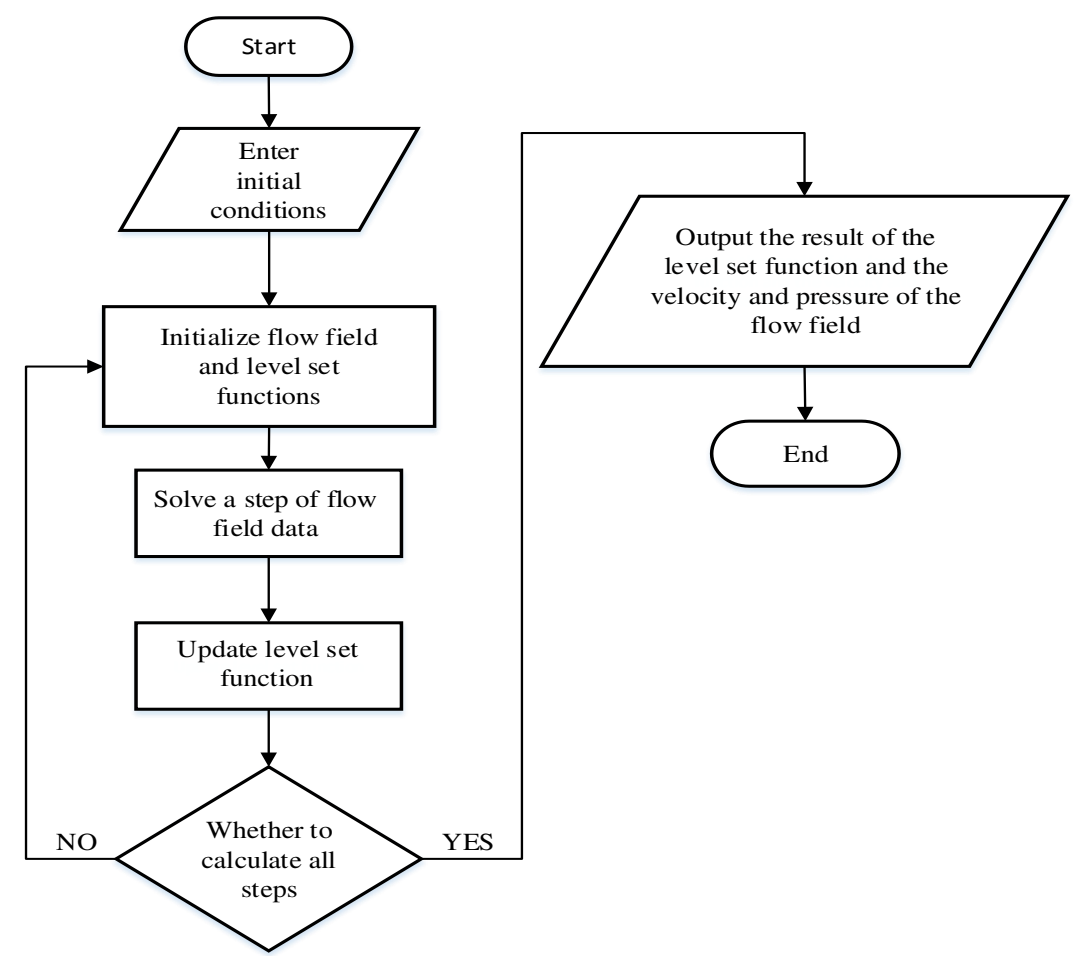

Fig. 6 Flow chart of bubble tracking calculation

\subsection{Calculation of discharge point position}

During the WEDM-LS, the place where the discharge breakdown occurs is definitely the place with the largest electric field strength. Therefore, when the interelectrode electric field strength is greater than the minimum breakdown electric field strength of deionized water, at this time, a breakdown discharge occurs between the electrode wire and the workpiece and electrical discharge processing begins. But it is different when there are bubbles in the discharge gap. The breakdown discharge occurs when either the electric field strength between the electrodes or the distortion electric field strength at the position of the bubble is greater than the minimum breakdown electric field strength of the deionized water.

Based on the above-mentioned discharge breakdown mechanism, the calculation process of discharge point position is shown below: Firstly, the shortest distance between the electrode wire and the workpiece is determined by calculating the vibration response of the electrode wire. The electric field strength there is obtained based on the voltage and the shortest distance at this time, then the position of the discharge point of the first discharge can be obtained. Set this position as the initial position of the bubble, and then the level set function is used to solve the bubble trajectory. In addition, the electric field strength at the position of the bubble can be calculated according to the electric field distortion formula. The calculated maximum electric field strength at the position of the bubble and the electric field intensity at the nearest point are compared with the breakdown electric field strength, and then the position of the discharge point can be determined and output. This is a time step. Afterwards, the solution is repeated until the last time step is solved, all the discharge point positions are output, and the calculation is finished. The schematic of the calculation of the discharge point is shown in Fig. 7. 


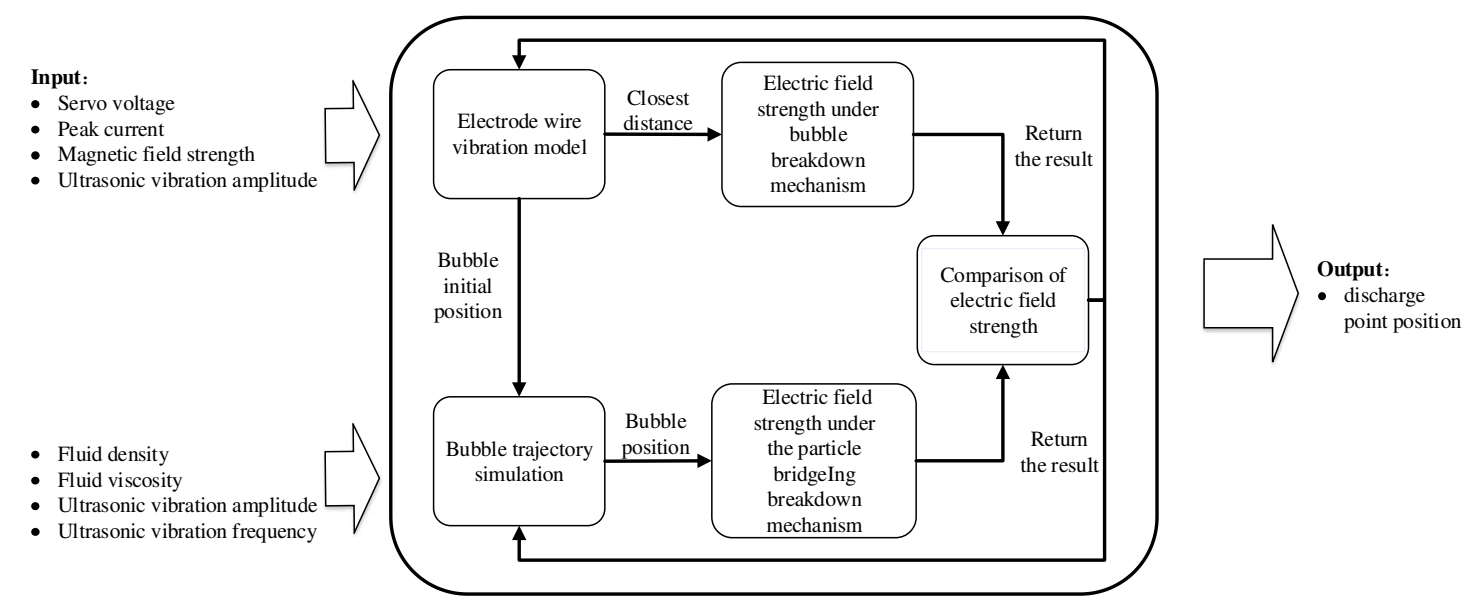

Fig. 7 Schematic of the calculation of discharge point

\section{The Model of discharge channel}

In this paper, a mathematical model is established to calculate the radius of the discharge channel. As shown in Fig. 8, a partial differential equation on the radius of the plasma channel can be obtained according to Newton's second law. From Eq. 25, it can be known that the radius of the discharge channel follows the movement state of electron in the discharge channel, the magnetic field around discharge channel, the internal and external pressure of the discharge channel, and surface tension at the interface [12].

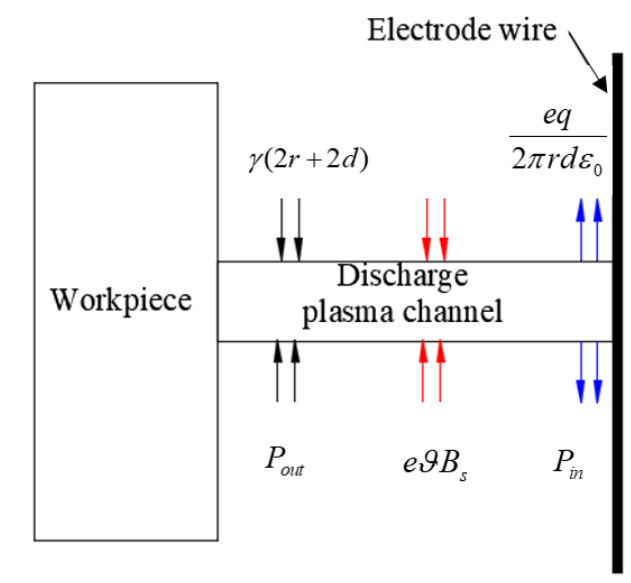

Fig. 8 Schematic diagram of the discharge channel

$$
\frac{d^{2} r}{d t_{\text {on }}{ }^{2}}=\frac{e q}{2 \pi r d \varepsilon_{0} m}-\frac{e \vartheta B_{s}}{m}+\frac{P_{\text {in }}(2 r d)}{m}-\frac{P_{\text {out }}(2 r d)}{m}-\frac{\gamma(2 r+2 d)}{m}
$$

Where, $r$ is the radius of the discharge channel, $t_{\text {on }}$ is the duration of the pulse, $e$ is the charge of the electrons, $q$ is the charge of the charged particles in the discharge channel, $d$ is the discharge gap distance, $\varepsilon_{0}$ is the vacuum permittivity, and $m$ is the mass of the particles in the discharge channel, $\vartheta$ is the drift speed of charged particles in the discharge channel, $B_{s}$ is the surrounding magnetic field generated by the movement of charged particles in the discharge channel, $P_{i n}$ is the pressure in the discharge channel, $P_{\text {out }}$ is the external pressure of the dielectric liquid on the discharge channel, and $\gamma$ is surface tension at the interface between the 
discharge channel and deionized water.

The pressure of deionized water on the discharge channel in Eq. (25) can be expressed as Eq. (26).

$$
P_{\text {out }}=P_{0}+\rho g h
$$

Where, $P_{0}$ is the standard atmospheric pressure, $\rho$ is the density of deionized water, $g$ is the gravitational acceleration, and $h$ is the distance of the discharge channel from the water surface.

Under the action of ultrasound, the pressure of the deionized water on the discharge channel can be expressed as Eq. (27).

$$
P_{\text {out }}{ }^{\prime}=P_{a c} \sin \omega t+P_{0}+\rho g h
$$

Where, $P_{a c}$ is the pressure disturbance of deionized water caused by the ultrasonic vibration, and $P_{a c}$ is defined as Eq. (28).

$$
P_{a c}= \pm 2 \pi f A \rho C_{s}
$$

Where, $f$ is the frequency of ultrasonic vibration, $A$ is the amplitude of ultrasonic vibration, $\rho$ is the density of deionized water, $C_{s}$ is the speed of sound in deionized water.

The surrounding magnetic field generated by the motion of the charged particle in the discharge channel in Eq. 25 is calculated as follows. Assuming that the radius of the discharge channel is a, the current density in the discharge channel is $j(r)$, the magnetic induction intensity is $B(r)$, the pressure distribution in the discharge channel is $\mathrm{P}(r)$, and $\mu_{0}$ is the magnetic permeability, then Maxwell's equation can be expressed in the cylindrical coordinate system as [13]:

$$
\frac{1}{r} \frac{\mathrm{d}}{\mathrm{d} r}[r B(r)]=\mu_{0} j(r)
$$

By solving Eq. (29), the magnetic field distribution in the discharge channel is

$$
B(r)=\frac{\mu_{0}}{r} \int_{0}^{r} r j(r) \mathrm{d} r
$$

Since the discharge channel is very narrow, it can be assumed that the current density $j$ inside the channel is uniformly distributed, and the magnetic field distribution in the discharge channel can be simplified as:

$$
B(r)=\frac{I \mu_{0} r}{2 \pi a^{2}}
$$

The magnetic field strength outside the discharge channel can be regarded as the surrounding magnetic field generated by the energized wire, which is expressed as: 


$$
B(r)=\frac{\mu_{0}}{r} \int_{0}^{r_{1}} r j(r) \mathrm{d} r=\frac{\mu_{0} I}{2 \pi r}
$$

Magnetic field strength can be calculated as

$$
\begin{cases}B_{s}=\frac{I \mu_{0} r}{2 \pi a^{2}} & (r<a) \\ B_{s}=\frac{\mu_{0} I}{2 \pi r} & (r>a)\end{cases}
$$

Under the external magnetic field, the discharge gap can be regarded as a crack in an infinitely large flat plate when calculating the actual magnetic field in the discharge gap. It shows that the external magnetic field can be distorted in the discharge gap flow field. Distortion caused by the external magnetic field is $B_{e}$ [14].

$$
B_{e}=\frac{\chi B_{0}}{\mu_{0} \mu_{\mathrm{r}}} \frac{(\kappa+1)}{4 G}
$$

Where, $B_{0}$ is the applied external magnetic field, $\mu_{0}$ is the permeability of deionized water, $\mu_{\mathrm{r}}$ is the permeability of workpiece, and $\chi 、 \kappa 、 G$ is the Lame parameter of the workpiece, The magnetic field strength around the discharge channel can be expressed as

$$
B_{p}=B_{e}+B_{s}
$$

Substituting Eq. (27) and Eq. (35) into Eq. (25), the model of the discharge channel under USV-MF assisted WEDM-LS is:

$$
\frac{d^{2} r}{d t_{o n}{ }^{2}}=\frac{e q}{2 \pi r d \varepsilon_{0} m}-\frac{e \vartheta B_{p}}{m}+\frac{P_{i n}(2 r d)}{m}-\frac{P_{o u t}{ }^{\prime}(2 r d)}{m}-\frac{\gamma(2 r+2 d)}{m}
$$

Namely:

$$
\begin{aligned}
\frac{d^{2} r}{d t_{o n}{ }^{2}}= & \frac{e q}{2 \pi r d \varepsilon_{0} m}-\frac{e \vartheta B_{e}+B_{s}}{m}+\frac{P_{i n}(2 r d)}{m}- \\
& \frac{P_{a c} \sin \omega t+P_{0}+\rho g h(2 r d)}{m}-\frac{\gamma(2 r+2 d)}{m}
\end{aligned}
$$

According to Eq. (37), the partial differential equation is calculated in MATLAB.

\section{Simulation analysis}

\subsection{The discharge position}

The discharge positions in the discharge channel was simulated by the Livelink Matlab module in COMSOL Multiphysics, and the module can perform joint calculations with Matlab. The number of discharges simulated in this paper is set to 500, and the simulation parameters are shown in Table 1. The simulation results are shown in Fig. 9. 
Table 1 Parameters used for simulation

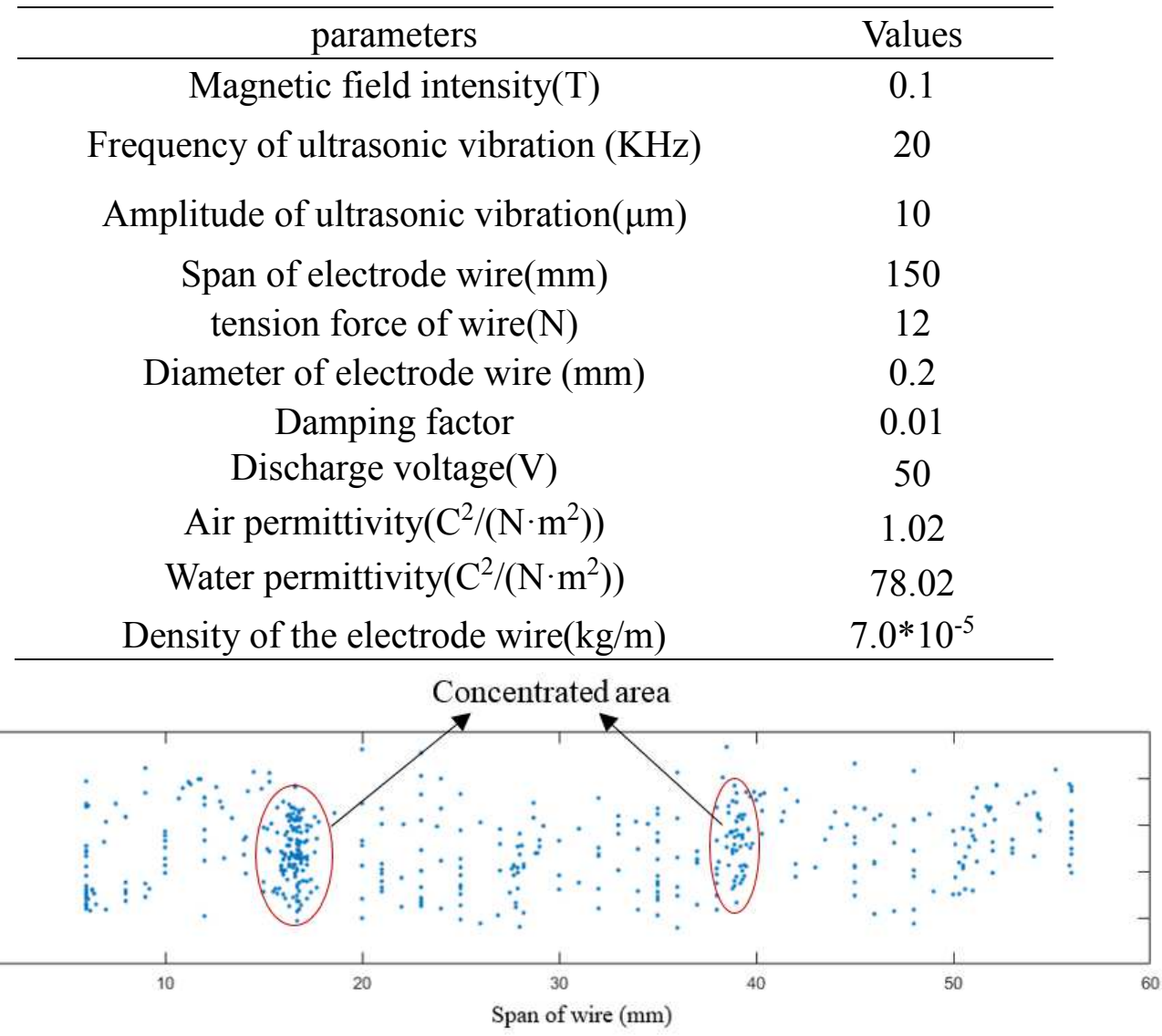

(a) Traditional WEDM-LS $\left(T_{\text {on }}=15 \mu \mathrm{s}, I P=6 \mathrm{~A}\right)$

(b)

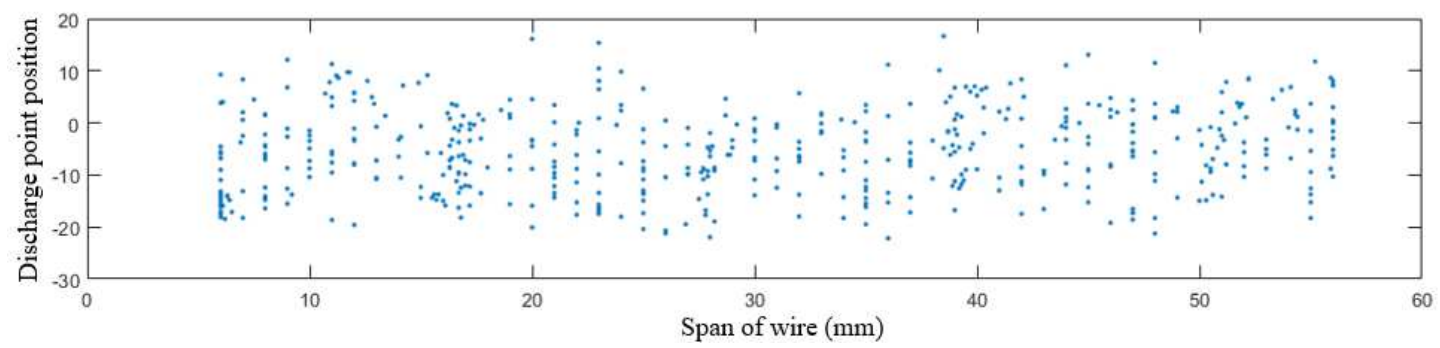

(c) USV-MF assisted WEDM-LS ( $\left.T_{\text {on }}=15 \mu \mathrm{s}, I P=6 \mathrm{~A}\right)$

Fig. 9 The distribution of discharge position in discharge channel

Fig. 9 is the distribution of discharge position in the machining area in the traditional WEDM-LS and the USV-MF assisted WEDM-LS under the same parameters. As shown in Fig. 9 (a), there are two areas where the discharge points are concentrated. This is because concentrated discharges are generated in two places on the workpiece surface due to the distortion of the inter-electrode electric field caused by bubbles in the traditional WEDM-LS. Concentrated discharge phenomenon causes the workpiece surface to be burnt, which has a great impact on the surface quality of the workpiece.

As shown in Fig. 9 (b), the distribution of discharge position becomes uniform under the application of ultrasonic vibration and external magnetic field, and the 
previous concentration area disappears. It shows that the bubbles in the discharge gap are broken by the effect of ultrasonic vibration, which reduces the distortion of the interelectrode electric field. The discharge between the electrode wire and the workpiece is mostly determined by the shortest distance discharge principle.

\subsection{The time-varying radius of discharge channel}

The partial differential equation in the model of the discharge channel was calculated in Matlab. The initial radius of the discharge channel $R_{0}$ and the initial pressure $P_{0}$ can be obtained by reference [15], and the calculation step is set to 0.001 $u s$. The results are shown in Table 2 .

Table 2 Results of the radius of discharge channel

(a) Traditional WEDM-LS

\begin{tabular}{ccc}
\multicolumn{3}{c}{$\left(T_{\text {on }}=15 \mu \mathrm{s}, I P=6 \mathrm{~A}\right)$} \\
\hline $\begin{array}{c}\text { Time } \\
(\mu \mathrm{s})\end{array}$ & $\begin{array}{c}\text { Radius } \\
(\mu \mathrm{m})\end{array}$ & $\begin{array}{c}\text { Pressure } \\
(\mathrm{MPa})\end{array}$ \\
\hline 0.000 & 0.081 & 6.11 \\
0.001 & 0.1075 & 5.50 \\
0.010 & 0.2605 & 2.02 \\
0.100 & 0.731 & 1.02 \\
1.000 & 2.6155 & 0.962 \\
2.000 & 4.264 & 0.842 \\
5.000 & 7.1075 & 0.752 \\
10.000 & 9.628 & 0.642 \\
13.000 & 10.7645 & 0.385 \\
14.000 & 11.128 & 0.385 \\
15.000 & 11.2665 & 0.385 \\
\hline
\end{tabular}

(b) USV-MF assisted WEDM-LS

\begin{tabular}{ccc} 
& \multicolumn{2}{c}{$\left(T_{\text {on }}=15 \mu \mathrm{s}, I P=6 \mathrm{~A}\right)$} \\
\hline $\begin{array}{c}\text { Time } \\
(\mu \mathrm{s})\end{array}$ & $\begin{array}{c}\text { Radius } \\
(\mu \mathrm{m})\end{array}$ & $\begin{array}{c}\text { Pressure } \\
(\mathrm{MPa})\end{array}$ \\
\hline 0.000 & 0.0915 & 6.031 \\
0.001 & 0.1575 & 5.232 \\
0.010 & 0.4105 & 1.954 \\
0.100 & 1.231 & 0.982 \\
1.000 & 3.6155 & 0.950 \\
2.000 & 6.264 & 0.825 \\
5.000 & 11.799 & 0.743 \\
10.000 & 13.551 & 0.521 \\
13.000 & 15.6195 & 0.365 \\
14.000 & 16.1265 & 0.365 \\
15.000 & 16.6065 & 0.365 \\
\hline
\end{tabular}

It can be seen from Table 2. (a) and Table 2. (b) that when the radius of discharge channel achieve equilibrium, the radius of discharge channel in USV-MF assisted WEDM-LS is 1.47 times that in traditional WEDM-LS, and the former takes longer to achieve equilibrium than the latter.

This is because the external magnetic field generates magnetic field perturbations on the discharge channel itself, forming a relatively weak magnetic field pinch effect around the discharge channel, which makes the oscillation of discharge channel strengthened. Coupled with the cavitation effect of ultrasonic vibration on the gap flow field, the compression effect of deionized water on the discharge channel is correspondingly reduced, resulting in longer expansion time of the discharge channel and finally an increase in the radius of discharge channel at equilibrium.

\subsection{The surface morphology of workpiece}

The surface morphology of workpiece under continuous discharge was simulated in the finite element software COMSOL Multiphysics. In the moving mesh module, it was set that the workpiece material will be removed automatically when the temperature reaches the melting point. The heat source is loaded by a Gaussian heat source [16], and the heat source loading equation is shown in Eq. (39). 


$$
q(r)=\frac{k \eta U I}{\pi R(t)^{2}} \exp \left(-k \frac{r^{2}}{R(t)^{2}}\right)
$$

Where, $q(r)$ is the heat flux density, $k$ is the heat flux concentration factor; $\eta$ is the energy distribution factor; $U$ is the discharge breakdown voltage; $I$ is the peak discharge current; $R(t)$ is the radius of the discharge channel.

$R(t)$ is selected through Table 2. The loading point of the heat source is set according to the discharge position coordinates in Fig. 9. The simulation results are shown in Fig. 10.

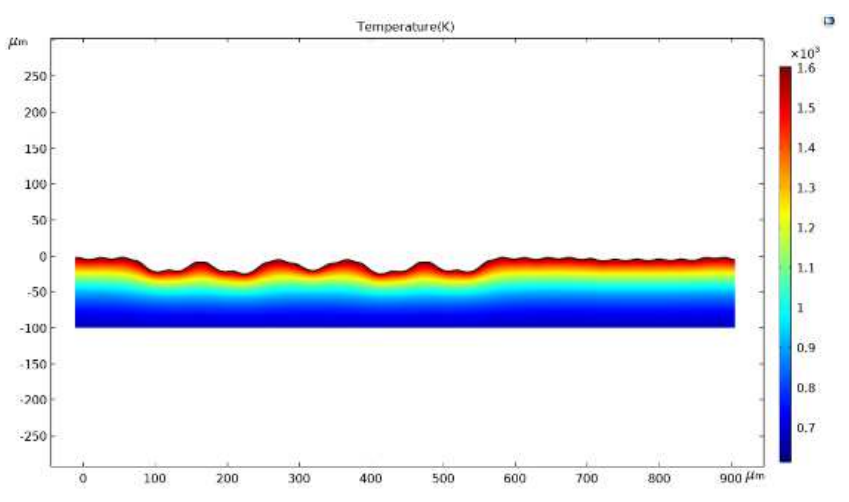

(a) Traditional WEDM-LS $\left(T_{\text {on }}=15 \mu \mathrm{s}, I P=10 \mathrm{~A}\right)$

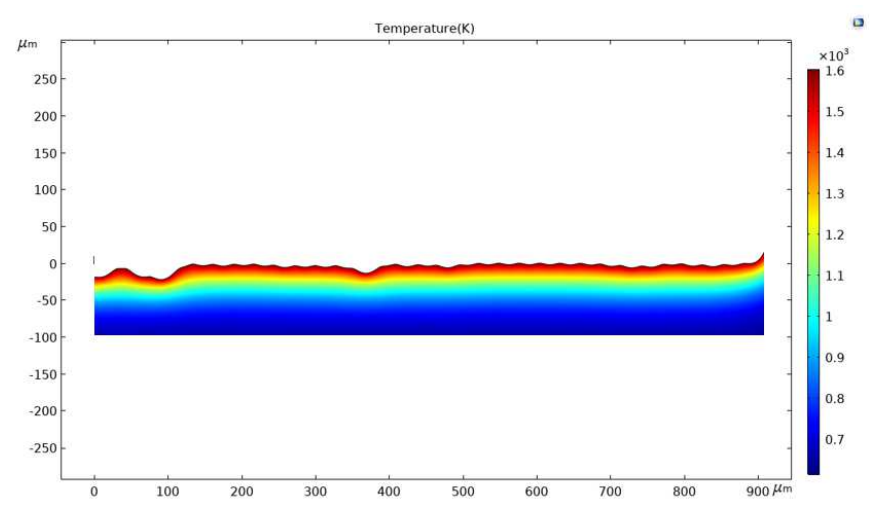

(b) USV-MF assisted WEDM-LS ( $T_{\text {on }}=15 \mu \mathrm{s}, I P=10 \mathrm{~A}$ )

Fig. 10 Surface morphology of workpiece under continuous discharge machining

\section{Experiment details}

\subsection{Experiment equipment}

In order to verify the correctness of the model, 12 sets of orthogonal tests were performed on SDK7625P machine tool. The test processing parameters are shown in Table 3. During the processing, the high-speed camera was used to capture the discharge points. When the oscilloscope detects that a current passes through the current loop, the oscilloscope sends a signal to the high-speed camera to start 
shooting, and the high-speed camera starts to capture. Finally, the machined workpiece is inspected by the testing instrument. The experiment setup is shown in Fig. 11.

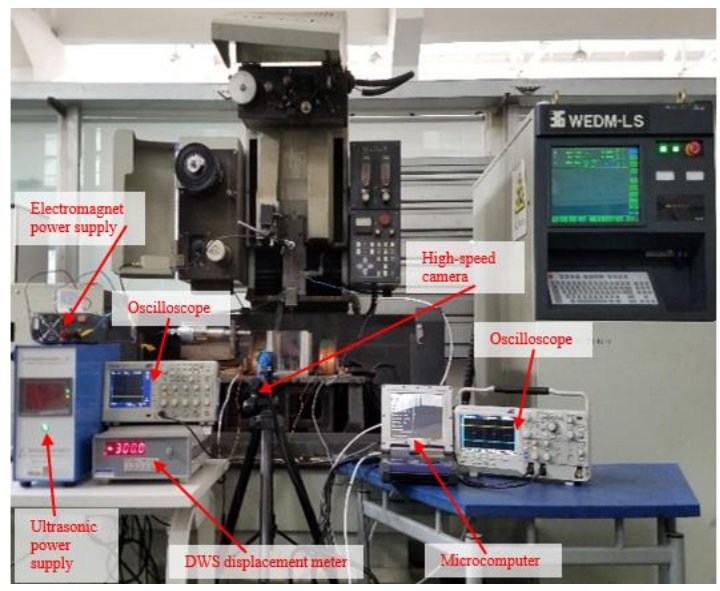

(a) Global photograph

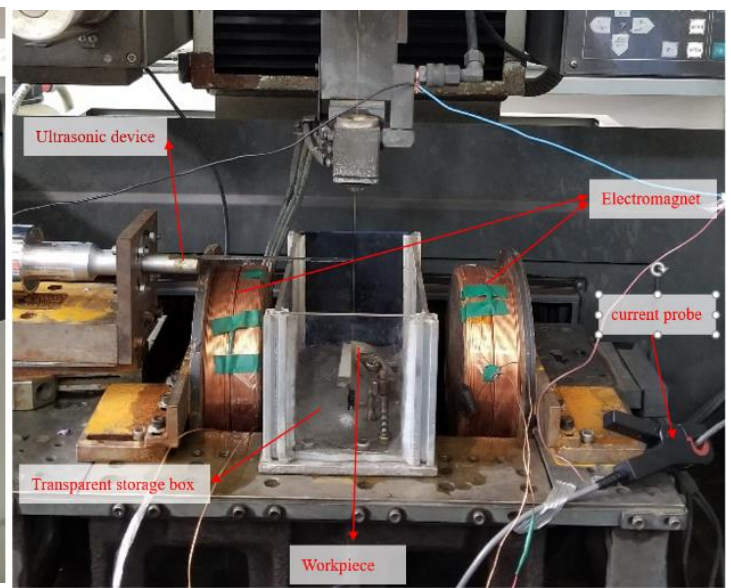

(b) Partial photograph

Fig. 11 Experimental setup

Table 3 Experimental parameters

\begin{tabular}{ll}
\hline Properties & Value \\
\hline Material & TiNi-01 \\
size/mm & $5 \times 3 \times 50$ \\
Magnetic field strength $/ \mathrm{T}$ & 0.1 \\
Ultrasound amplitude & 10 \\
$/ \mu \mathrm{m}$ & 15 \\
Pulse width / $\mu \mathrm{s}$ & 10000 \\
Shooting frequency /fps & $6,8,10$ \\
Discharge current /A & \\
\hline
\end{tabular}

\subsection{Experimental results and discussion}

\subsubsection{The distribution of discharge points}

The shooting frame rate of the high speed camera was set to $10000 \mathrm{fps} / \mathrm{s}$, and the captured images were sorted according to the shooting order as shown in Fig. 12 and Fig. 13.

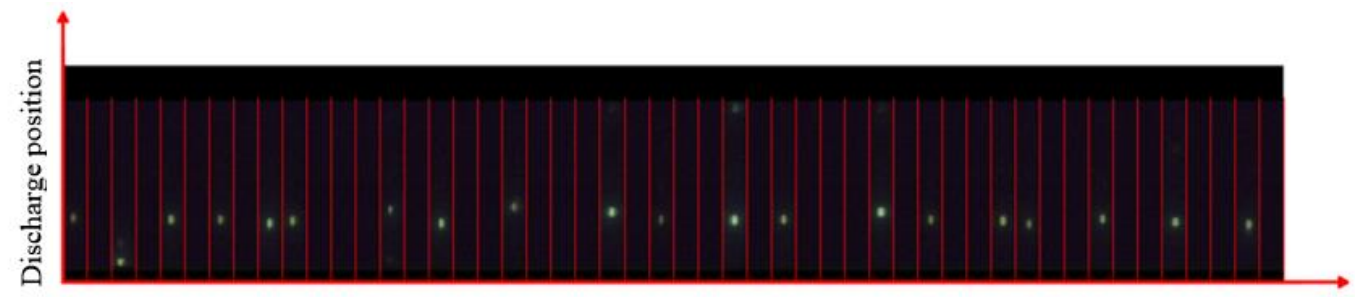

The serial number of the picture

(a) 0-50fps 


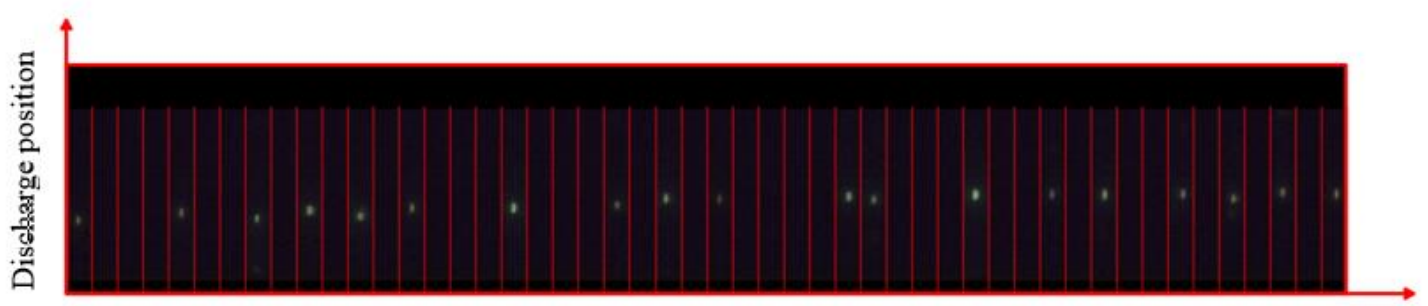

The serial number of the picture

(b) 51-100fps

Fig. 12 Distribution of discharge points in traditional WEDM-LS

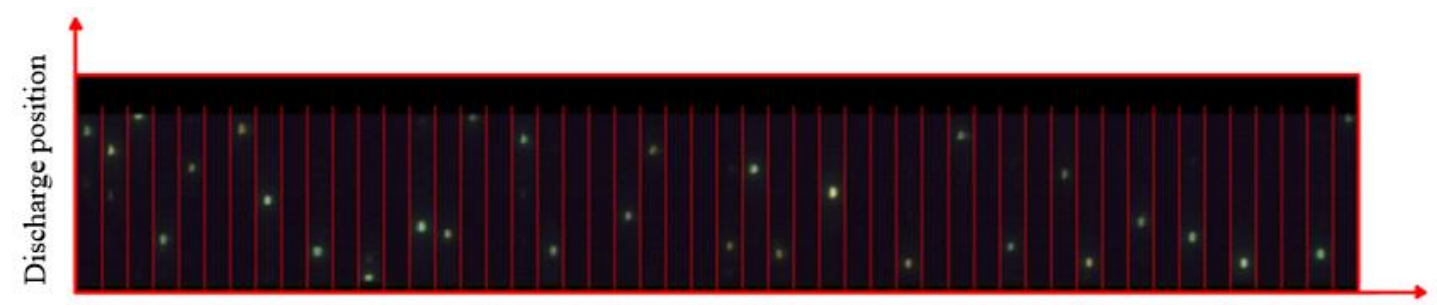

The serial number of the picture

(a) 0-50fp

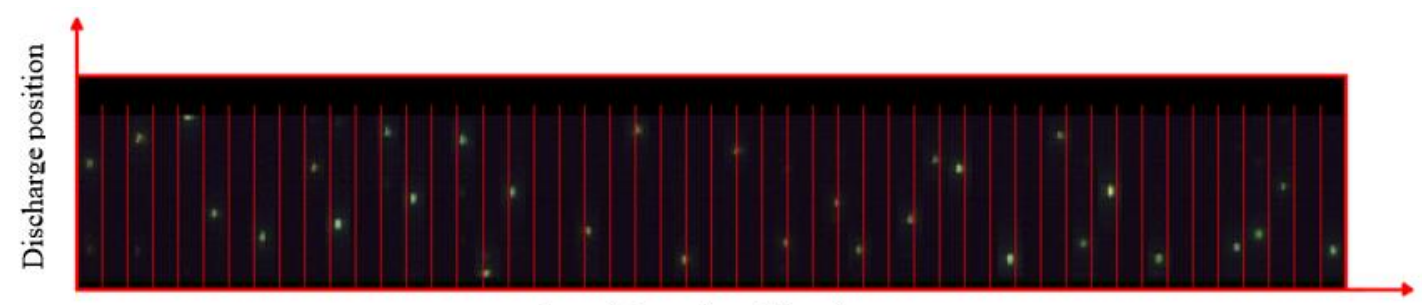

The serial number of the picture

(b) 51-100fps

Fig. 13 Distribution of discharge points in USV-MF assisted WEDM-LS

Fig. 12 are the distribution of discharge points in traditional WEDM-LS. Due to the distortion effect of bubbles on the inter-electrode field, discharge points are centrally distributed in a certain area on the workpiece surface, and a concentrated discharge phenomenon occurs there during the time of 0-100 frames. And in some frames of the picture, the discharge point was not captured, which means that the discharge was not successful at that moment. By sorting the results of the discharge points, the discharge success rate of traditional WEDM-LS was obtained 41.2\%. Fig. 13 are the distribution of discharge points in USV-MF assisted WEDM-LS. In 0-100 frames, the discharge points are scattered on the workpiece surface, which reduces the occurrence of concentrated discharge and the discharge success rate was increased to $62.2 \%$. It is shown that the USV-MF assisted WEDM-LS can reduce the occurrence of concentrated discharge during the WEDM and increase the discharge success rate.

The Christiansen uniformity coefficient $U$ was used to evaluate the uniformity of discharge points, and it can be calculated as follows:

$$
U=100 \% \times\left[1-\frac{\sum_{i=1}^{N}\left|p_{i}-\bar{p}\right|}{N \bar{p}}\right]
$$

Where $N$ is the number of test areas, $p_{i}$ is the number of discharge points in the area $i$ and $\bar{p}$ is the average number of discharge points. 
The captured pictures of discharge points in traditional WEDM-LS and USV-MF assisted WEDM-LS were divided into 10 areas along the $Y$ axis, and the number of discharge points can be obtained in each area, respectively. The uniformity coefficient under different machining parameters can be calculated as shown in Fig.14.

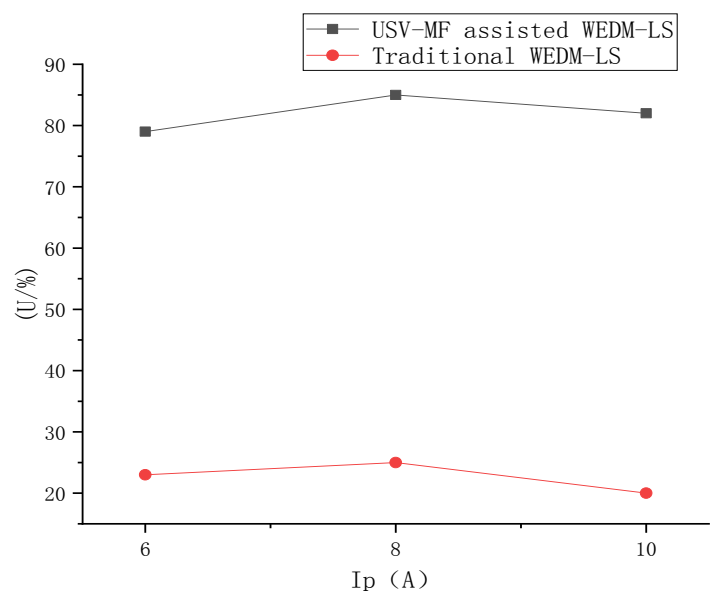

Fig. 14 The uniformity coefficient under different machining parameters

It can be seen that under the same machining parameters, the uniformity coefficient in USV-MF assisted WEDM-LS is higher than that in traditional WEDMLS. Meanwhile, the uniformity coefficient changes little with the change of machining parameters. The uniformity coefficient in USV-MF assisted WEDM-LS is about $80 \%$ while that is about $22 \%$ in traditional WEDM-LS, it indicates obviously that the distribution of discharge points in USV-MF assisted WEDM-LS is more uniform.

\subsubsection{Analysis of discharge waveforms}

The discharge waveform of traditional WEDM-LS and USV-MF assisted WEDMLS are shown in Fig. 15.

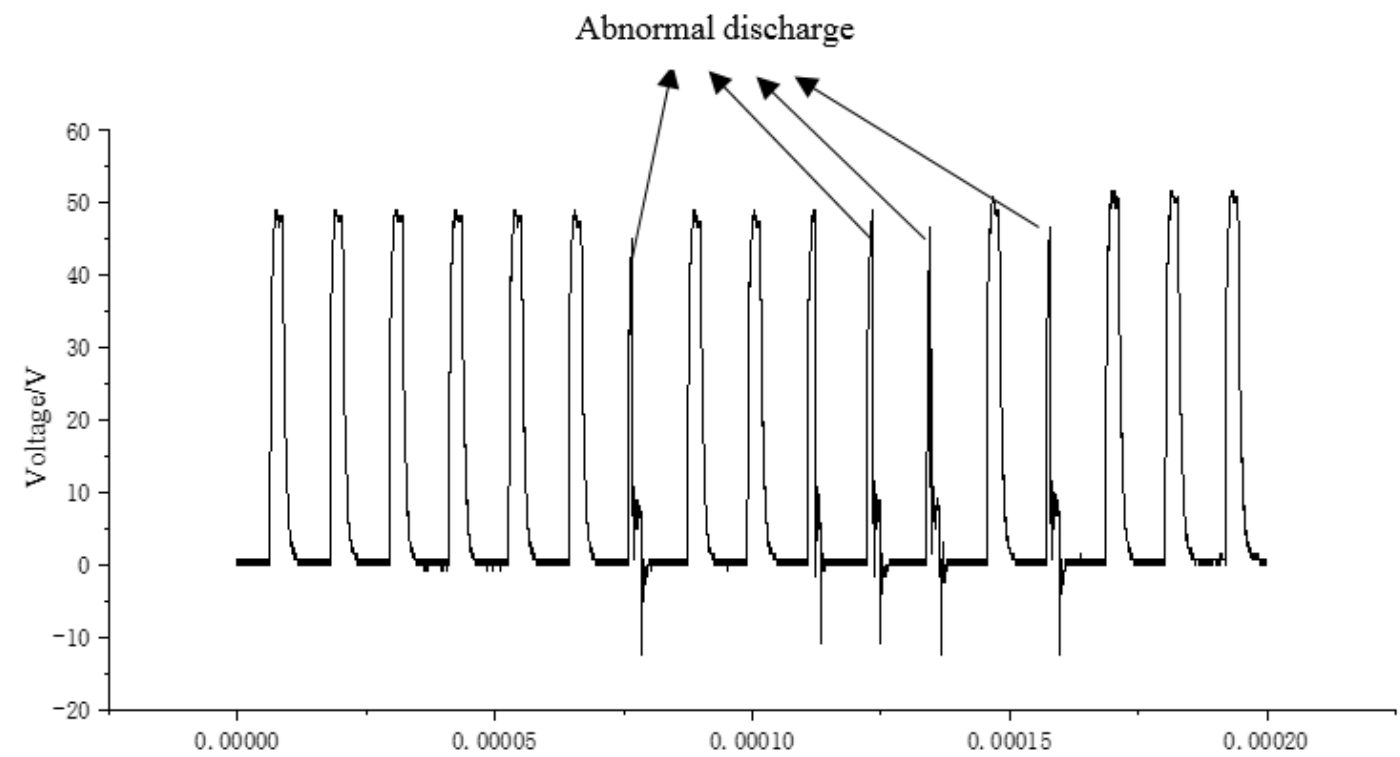

(a) Traditional WEDM-LS 


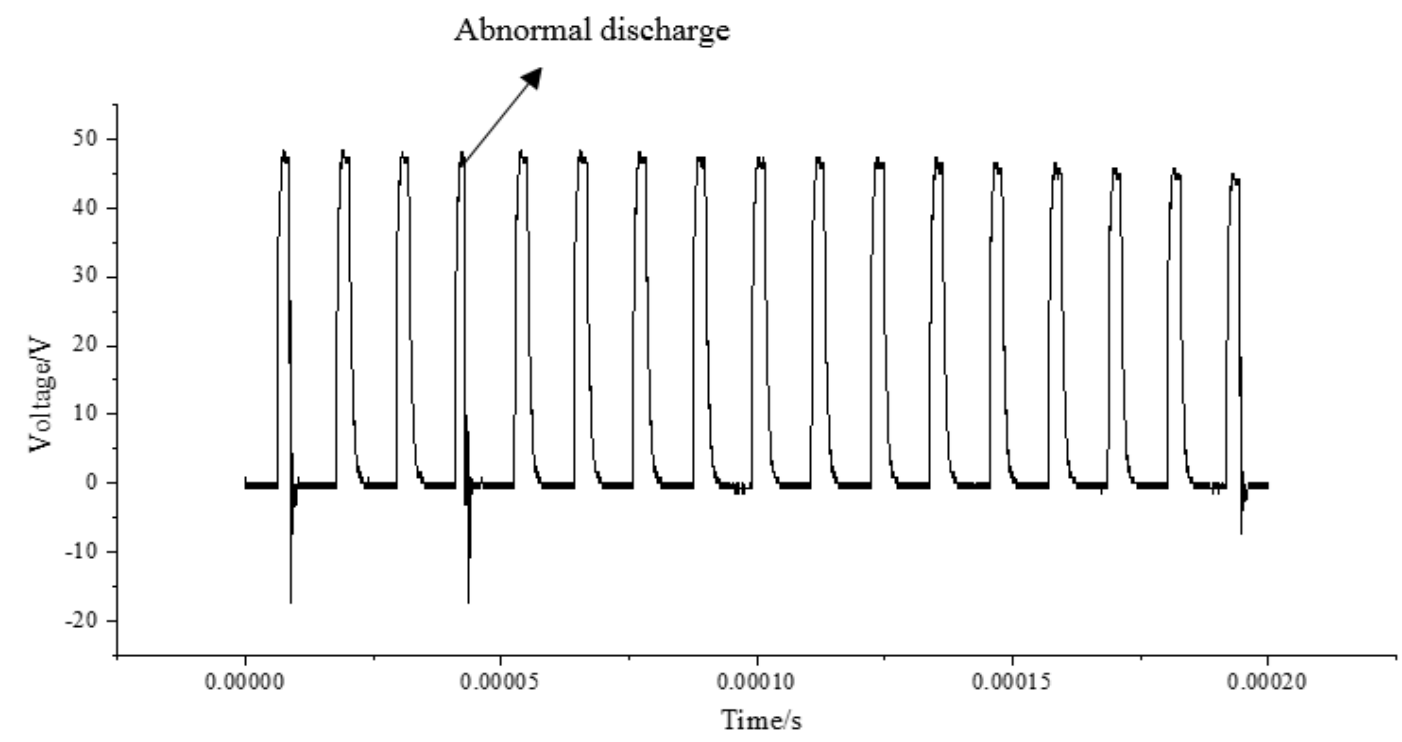

(d) USV-MF assisted WEDM-LS

Fig. 15 Discharge waveform

It can be known from Fig. 15 that there are many abnormal discharges in traditional WEDM-LS, which is significantly alleviated in USV-MF assisted WEDM-LS. The main reason is that the electrode wire is vibrated at a low frequency irregularly in traditional WEDM-LS, and the bubbles and corrosion products in the processing area cannot be expelled in time, leading to the failure of dielectric liquid to recover its dielectric properties in a short time, as a result, it is easy to cause abnormal discharge phenomena such as short circuit. However, the high-frequency sinusoidal vibration of electrode wire can increase the velocity of the working fluid and make the bubble burst in USV-MF assisted WEDM-LS. Thus, it improves the fluidity of the working fluid and speeds up the deionizing velocity of working fluid, which can avoid effectively the occurrence of abnormal discharge.

\subsubsection{Surface roughness value $R_{a}$}

The surface roughness of the workpiece was measured by a TAYLOR HOBSON roughness tester. The test results obtained are shown in Table 4.

Table 4 Experimental data

\begin{tabular}{ccccccc}
\hline NO. & $\begin{array}{c}\text { ton } \\
(\mu \mathrm{s})\end{array}$ & $\begin{array}{c}\text { toff } \\
(\mu \mathrm{s})\end{array}$ & $\begin{array}{c}\text { Ip } \\
(\mathrm{A})\end{array}$ & $\begin{array}{c}\text { Amplitude of } \\
\text { ultrasonic } \\
\text { vibration }(\mu \mathrm{m})\end{array}$ & $\begin{array}{c}\text { Magnetic } \\
\text { field } \\
\text { intensity(T) }\end{array}$ & $\begin{array}{c}\mathrm{Ra} \\
(\mu \mathrm{m})\end{array}$ \\
\hline 1 & 15 & 25 & 6 & 0 & 0 & 3.21 \\
2 & 15 & 25 & 6 & 10 & 0 & 2.34 \\
3 & 15 & 25 & 6 & 0 & 0.1 & 2.84 \\
4 & 15 & 25 & 6 & 10 & 0.1 & 1.98 \\
5 & 15 & 25 & 8 & 0 & 0 & 3.32 \\
6 & 15 & 25 & 8 & 10 & 0 & 2.65 \\
7 & 15 & 25 & 8 & 0 & 0.1 & 3.01 \\
8 & 15 & 25 & 8 & 10 & 0.1 & 2.23 \\
9 & 15 & 25 & 10 & 0 & 0 & 3.62
\end{tabular}




\begin{tabular}{ccccccc}
10 & 15 & 25 & 10 & 10 & 0 & 2.95 \\
11 & 15 & 25 & 10 & 0 & 0.1 & 3.21 \\
12 & 15 & 25 & 10 & 10 & 0.1 & 2.51 \\
\hline
\end{tabular}

Through a comparative analysis of the data in Table 4 , it can be obtained that as the peak voltage (Ip) increases, the surface roughness value of workpiece increases when the other parameters are the same. From the 6 sets of tests, in which the ultrasonic vibration and the magnetic field are applied separately in Table 4, it can be seen that both the ultrasonic vibration and the magnetic field can reduce the surface roughness value of workpiece, meanwhile, the ultrasonic vibration has a greater effect on that. From the overall analysis, it can be seen that the simultaneous addition of ultrasonic vibration and magnetic field has a better effect on reducing the surface roughness value and improves the machining quality of WEDM-LS.

\subsubsection{Crater diameter}

3D Electron Microscope VHX-7100 was used to detect crater diameter of the machined workpiece, and the magnification was 3000 times. The crater diameter image was obtained as shown in Fig. 16.

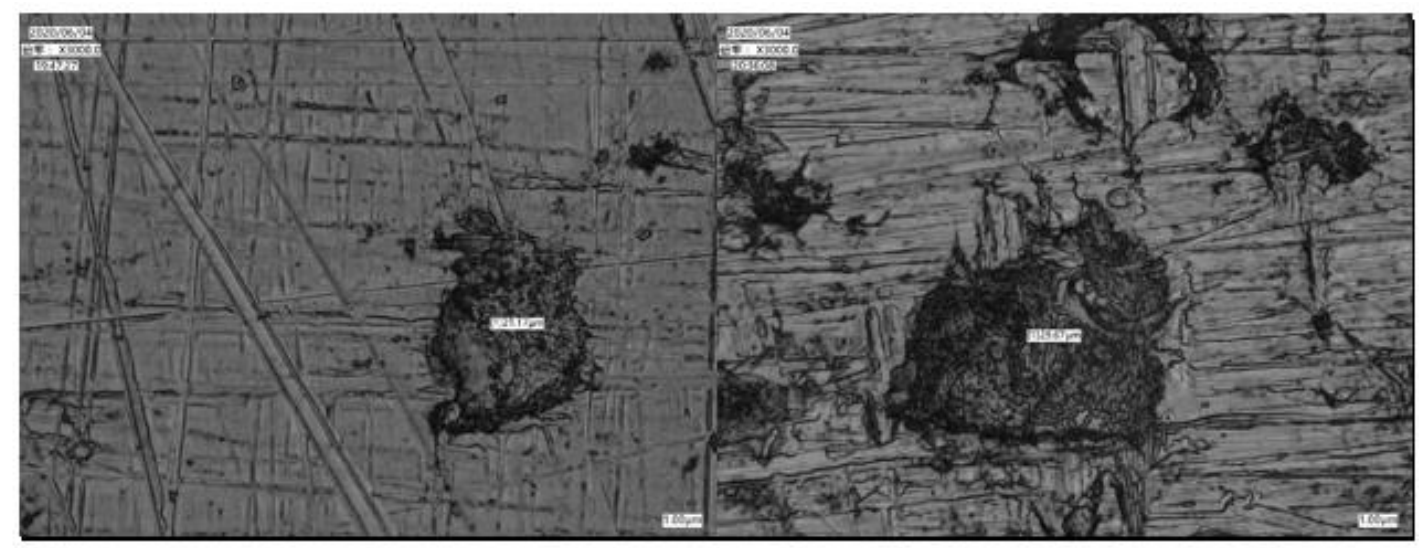

(a) Traditional WEDM-LS

(b) USV-MF assisted WEDM-LS

Fig. 16 Crater diameter of workpiece $\left(T_{\text {on }}=15 \mu \mathrm{s}, I P=10 \mathrm{~A}\right)$

It can be seen from Fig. 16 that the crater diameter of Traditional WEDM is $21.17 \mu \mathrm{m}$ and the crater diameter of USV-MF assisted WEDM-LS is $29.67 \mu \mathrm{m}$ under the same parameters. Comparing the calculated data in Table 2, the error between the simulated value and the measured value in Traditional WEDM-LS is $6.4 \%$, and the error between the simulated value and the measured value in USV-MF assisted WEDMLS is $10.7 \%$. Within a certain error range, it indicates the accuracy of the discharge channel model.

\subsubsection{D Surface morphology of workpiece}

The burns on the workpiece surface can be clearly seen by observing the machined workpiece, as shown in Fig. 17. 


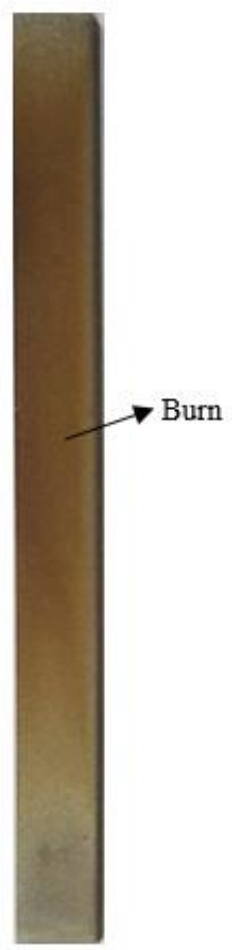

(a) Traditional WEDM-LS

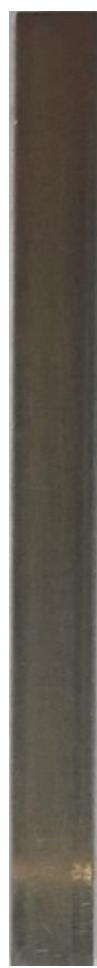

(b) USV-MF assisted WEDM-LS

Fig. 17 Workpiece surfaces machined in traditional WEDM-LS and USV-MF assisted WEDM-LS ( $\left.T_{\text {on }}=15 \mu \mathrm{s}, I P=10 \mathrm{~A}\right)$

It can be seen from Fig. 17 that the surface of the machined workpiece is seriously burned in traditional WEDM-LS because of the large thickness of workpiece, which affects the surface quality of the workpiece. However, there are almost no burned areas on the machined workpiece in USV-MF assisted WEDM-LS, which indicates that the compound machining can effectively improve the surface quality of the workpiece. In order to further analyze the influence of different machining conditions on the surface of the workpiece, the microstructure was analyzed and studied.

VHX-7100 was used to detect the 3D surface morphology of the machined workpiece, and the magnification was 1000 times. The 3D surface morphology image was obtained as shown in Fig. 18.

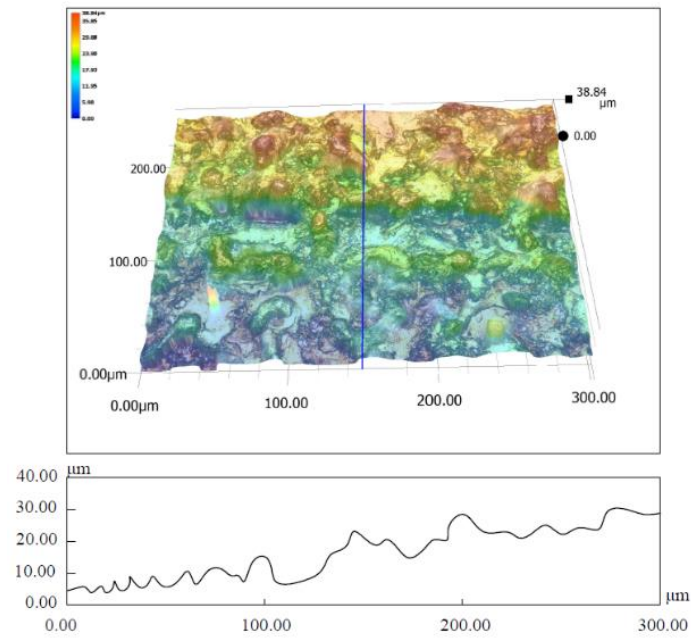

(a) Traditional WEDM-LS

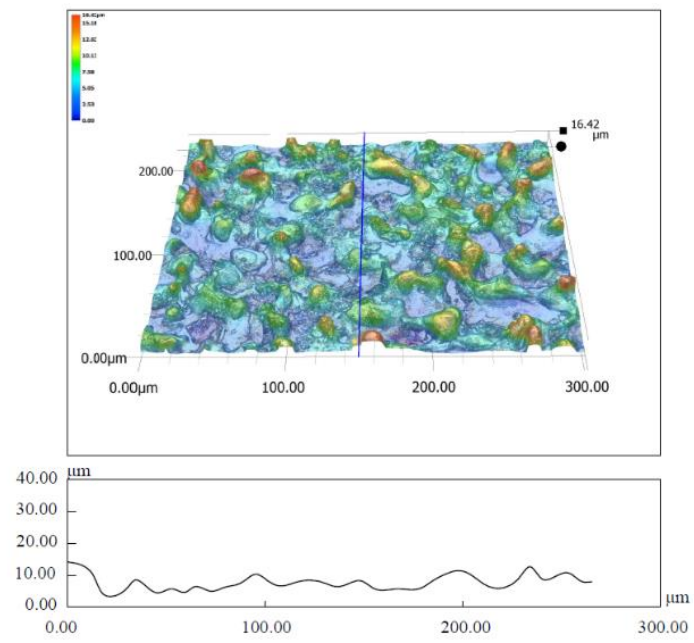

(b) USV-MF assisted WEDM-LS 
Fig. 18 3D surface morphology of workpiece $\left(T_{\text {on }}=15 \mu \mathrm{s}, I P=10 \mathrm{~A}\right)$

It can be seen from Fig. 18 that under the same parameters, the peak value of the surface morphology under USV-MF assisted WEDM-LS is $16.42 \mu \mathrm{m}$ and the peak value of that under traditional WEDM-LS processing is $38.84 \mu \mathrm{m}$, which is 2.37 times the former. Through the section profile curve, it can be seen that there is a sudden change in the height direction of the curve under traditional WEDM-LS, while the curve under USV-MF assisted WEDM-LS changes within a certain range in the height direction. It is verified that the concentrated discharge on a part of the workpiece surface increases the amount of erosion in that area, which increases the peak value of the surface morphology and causes burns to the workpiece surface.

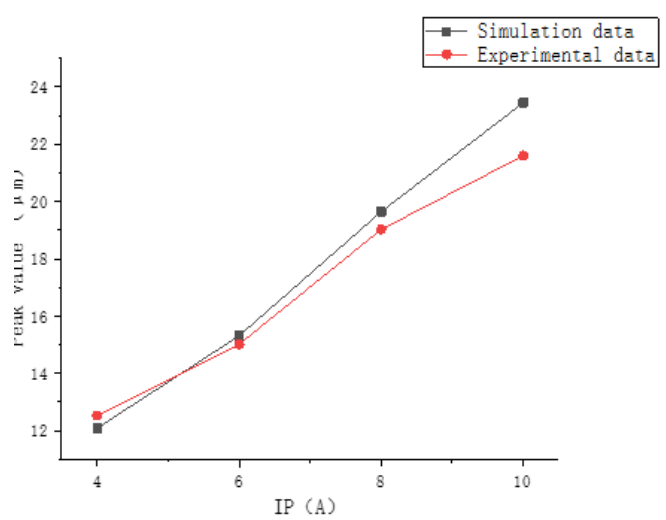

(a) Traditional WEDM-LS

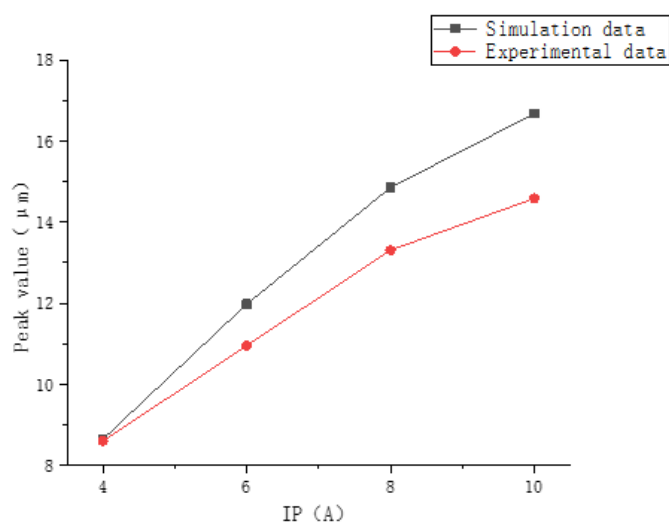

(b) USV-MF assisted WEDM-LS

Fig. 19 Experimental and simulated surface peak values

By comparing Fig.19 (a) and Fig.19 (b), it can be seen that the surface peak values of each group in traditional WEDM-LS is significantly larger than those in USV-MF assisted WEDM-LS and the maximum value of surface peak in traditional WEDMLS is $25 \mu \mathrm{m}$, while the maximum value of surface peak in USV-MF assisted WEDMLS is $17 \mathrm{um}$. The surface peak value increases with the increase of peak current. Because a large peak current means a large energy in a single discharge, the depth of crater produced by single discharge increases and the surface peak value obtained under continuous discharge also increases accordingly. It can be obtained from both Fig. 19 (a) and Fig. 19 (b) that the variation trend between the simulation value and the experimental value is the same in traditional WEDM-LS and USV-MF assisted WEDM-LS. The error between the simulation value and the experimental value in traditional WEDM-LS is $5.6 \%$ and that in USV-MF assisted WEDM-LS is $11.5 \%$, which indicates that the surface roughness can be predicted precisely by the simulation model.

\section{Conclusion}

In this paper, the shape and position characteristics of discharge channel in USVMF assisted WEDM-LS are studied and compared with that in traditional WEDM-LS. The effects of ultrasonic vibration and magnetic fields on the discharge channel are analyzed. The contrast experiment of traditional WEDM-LS and USV-MF assisted WEDM-LS workpiece with large thickness is used to verify the proposed theoretical 
model. The main conclusions are as follows:

(1) The bubbles generated in the WEDM-LS process will distort the electric field between the electrodes, resulting in the phenomenon of concentrated discharge. In order to explain this phenomenon, a mechanism of particle bridging breakdown suitable for machining workpiece with large thickness is proposed, and based on this mechanism, a model for predicting the discharge position of plasma discharge channels is established.

(2) From the results of the radius of discharge channel, it can be concluded that the radius of discharge channel in USV-MF assisted WEDM-LS is 1.47 times that in traditional WEDM-LS when it is at equilibrium. Considering that the erosion volume of a single discharge is the same, the diameter of the pits removed in USV-MF assisted WEDM-LS is large and the depth is shallow, and the surface quality of workpiece is significantly improved. Starting from the theoretical formula, the specific data of the discharge channel at each time point is of great significance for guiding the composite processing.

(3) By comparing the experimental results with the simulation results, it can be concluded that the distribution of the discharge points obtained by the simulation is basically consistent with that recorded in the experiment. The error between the peak value of the simulated surface morphology of workpiece under continuous discharge and that obtained from the experiment is within $6 \%$. It shows that the prediction model can well predict the surface morphology of workpiece with large thickness in USV-MF assisted WEDM-LS.

\section{Acknowledgement}

The authors thank the Natural Science Foundation of Shanghai for supporting for the study.

\section{Declarations}

Funding No funding was received for conducting this study.

Authors Contributions Yan Wang: Conceptualization, Methodology, Supervision, Writing- Review \& Editing. Jie Yang: Formal analysis, Visualization, Writing-Original Draft. Wei Xiong: Resources, Data curation. Huayi Chai: software, Data curation. Rui Wang: Visualization, software.

Data availability The manuscript has no associated data or the data will not be deposited.

Conflict of interest The authors declare that they have no competing interests.

Ethical approval This work has not been published elsewhere.

Consent to Participate Not applicable.

Consent to publish Not applicable. 


\section{References}

[1] Abbas NM, Solomon DG, Bahari MF (2007) A review on current research trends in electrical discharge machining (EDM). Int J Mach Tool Manuf 47:214-1228. https://doi.org/10.1016/j.ijmachtools.2006.08.026.

[2] Goiogana M, Sarasua JA, Ramos JM, Echavarri L, Cascon I (2016) Pulsed ultrasonic assisted electrical discharge machining for finishing operations. Int J Mach Tool Manuf 109:87-93. https://doi.org/10.1016/j.ijmachtools.2016.07.005.

[3] Chen Z, Zhang YM, Zhang GJ, Huang Y, Liu CH (2017) Theoretical and experimental study of magnetic-assisted finish cutting ferromagnetic material in WEDM. Int $\mathrm{J}$ Mach Tool Manuf 123:36-47. https://doi.org/10.1016/j.ijmachtools.2017.07.009.

[4] Wang Y, Wang Q, Ding Z (2018) Study on the mechanism and key technique of ultrasonic vibration and magnetic field assisted WEDM-LS thick shape memory alloy workpiece. J Mater Process Technol 261:251-265. https://doi.org/10.1016/j.jmatprotec.2018.06.006.

[5] Dhanik S, Joshi SS (2005) Modeling of a Single Resistance Capacitance Pulse Discharge in Micro-Electro Discharge Machining. J Manuf Sci Eng 127:759-767. https://doi.org/10.1115/1.2034512.

[6] Chu XY, Zhang K, Wang CM (2016) A Study on Plasma Channel Expansion in Micro-EDM. Mater Manuf Process 31:381-390. https://doi.org/10.1080/10426914.2015.1059445.

[7] Heuvelman HJ, Horsten JA, Veenstra PC (1971) An Introductory Investigation of Breakdown Mechanism in Electro-Discharge Machining. CIRP Ann 20:43-44. https://doi.org/10.1160/TH14-09-0721.

[8] Sauers I, James R, Ellis A (2009) Breakdown in Liquid Nitrogen in the Presence of Thermally Generated Bubbles for Different Electrode Geometries. IEEE 2009 Annual Report Conference on Electrical Insulation and Dielectric Phenomena. https://doi.org/10.1109/CEIDP.2009.5377752.

[9] Guo ZN, Lee TC, Yue TM, Lau WS (1997) A Study of Ultrasonic-aided Wire Electrical Discharge Machining. J Mater Process Technol 63:823-828. https://doi.org/10.1016/S0924-0136(96)02732-X.

[10] Tavakoli R, Babaei R, Varahram N, Davami P (2006) Numerical simulation of liquid/gas phase flow during mold filling. Comput Meth Appl Mech Eng 196:697713. https://doi.org/10.1016/j.cma.2006.06.001.

[11] Chorin AJ (1968) Numerical solution of the Navier-Stokes equations. Math Comput 22:745-762. https://doi.org/10.1090/S0025-5718-1968-0242392-2.

[12] Shabgard MR, Gholipoor A, Mohammadpourfard M (2019). Investigating the effects of external magnetic field on machining characteristics of electrical discharge machining process, numerically and experimentally. Int J Adv Manuf Technol 102:55-65. https://doi.org/10.1007/s00170-018-3167-3.

[13] Sen SN, Das RP (1973) Effect of magnetic field on primary ionization by electron collision. Proc Indian Natl Sci Acad, Part A;(India) 39(6).

[14] Liang W, Shen YP, Zhao M (2000) Magnetoelastic formulation of soft 
ferromagnetic elastic problems with collinear cracks: energy density fracture criterion. Theor Appl Fract Mech. https://doi.org/10.1016/S0167-8442(00)00023-9. [15] Patel MR, Barrufet MA, Eubank PT, DiBitonto DD (1989) Theoretical models of the electrical discharge machining process. II. The anode erosion model. J Appl Phys 66:4104-4111. https://doi.org/10.1063/1.343995.

[16] Yadav V, Jain VK, Dixit PM (2002) Thermal stresses due to electrical discharge machining. Int J Mach Tool Manuf 42:877-888. https://doi.org/10.1016/s08906955(02)00029-9. 
Figures
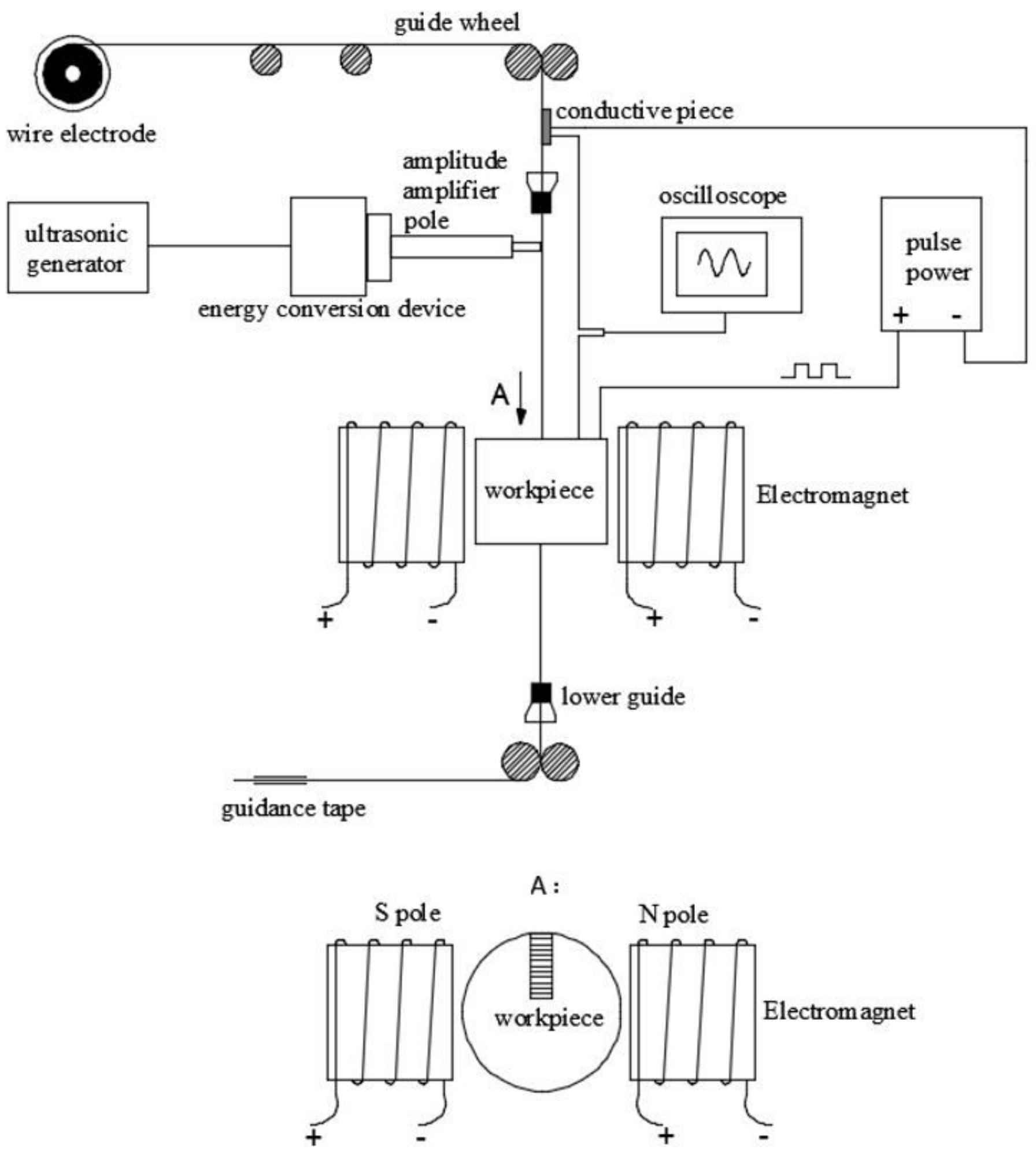

Figure 1

Schematic of USV-MF assisted WEDM-LS system 


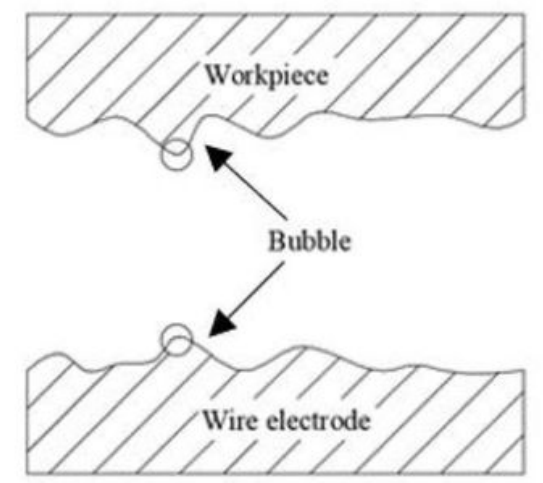

(a) Pre-breakdown state

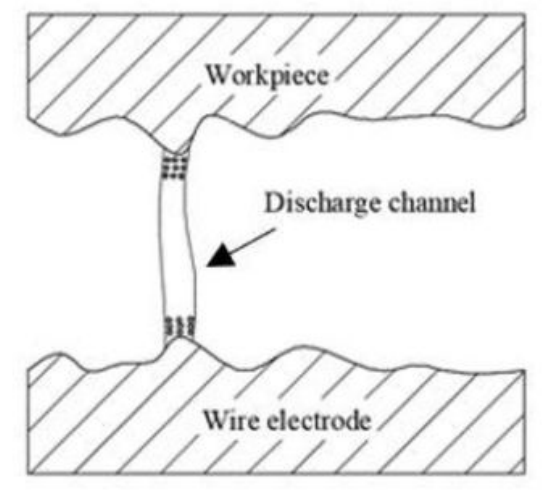

(b) Initial state

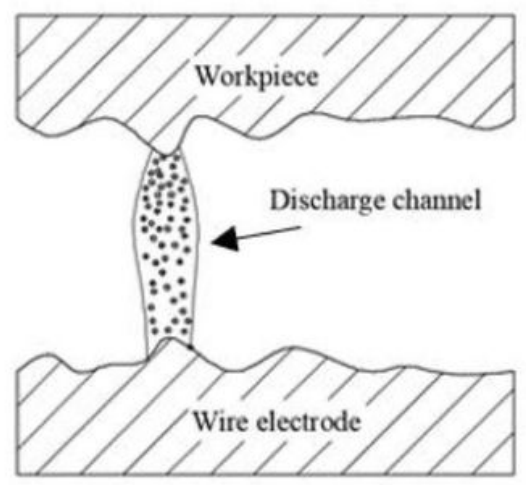

(c) Balance state

Figure 2

The formation process of the discharge channel under the bubble breakdown mechanism

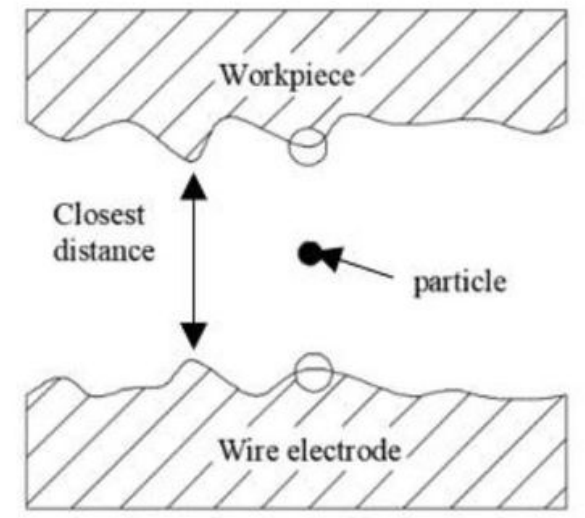

(a) Pre-breakdown state

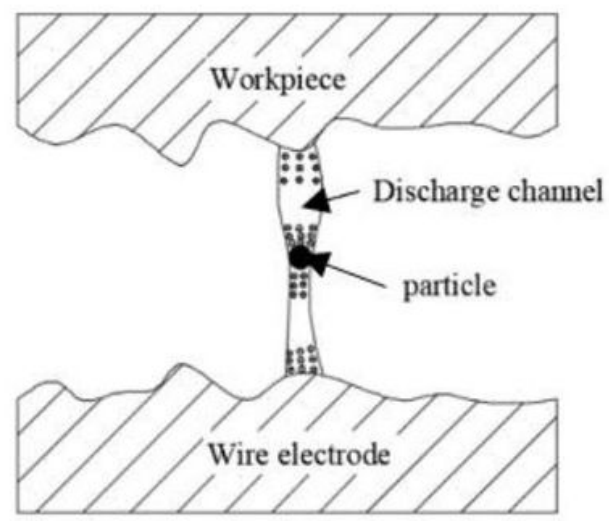

(b) Initial state

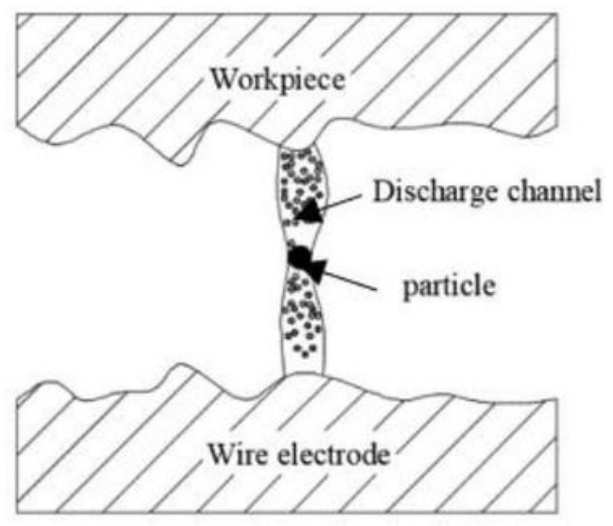

(c) Balance state

\section{Figure 3}

The formation process of the discharge channel of the "particle" bridge breakdown mechanism
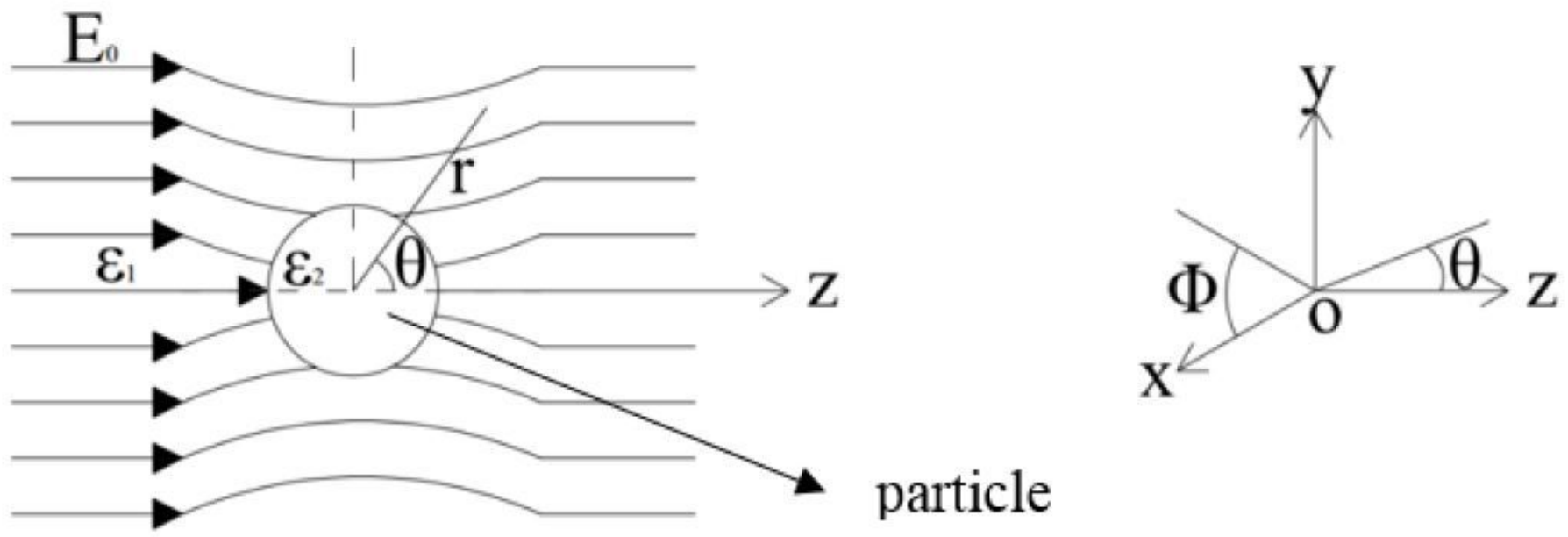

\section{Figure 4}


Schematic of electric field distortion

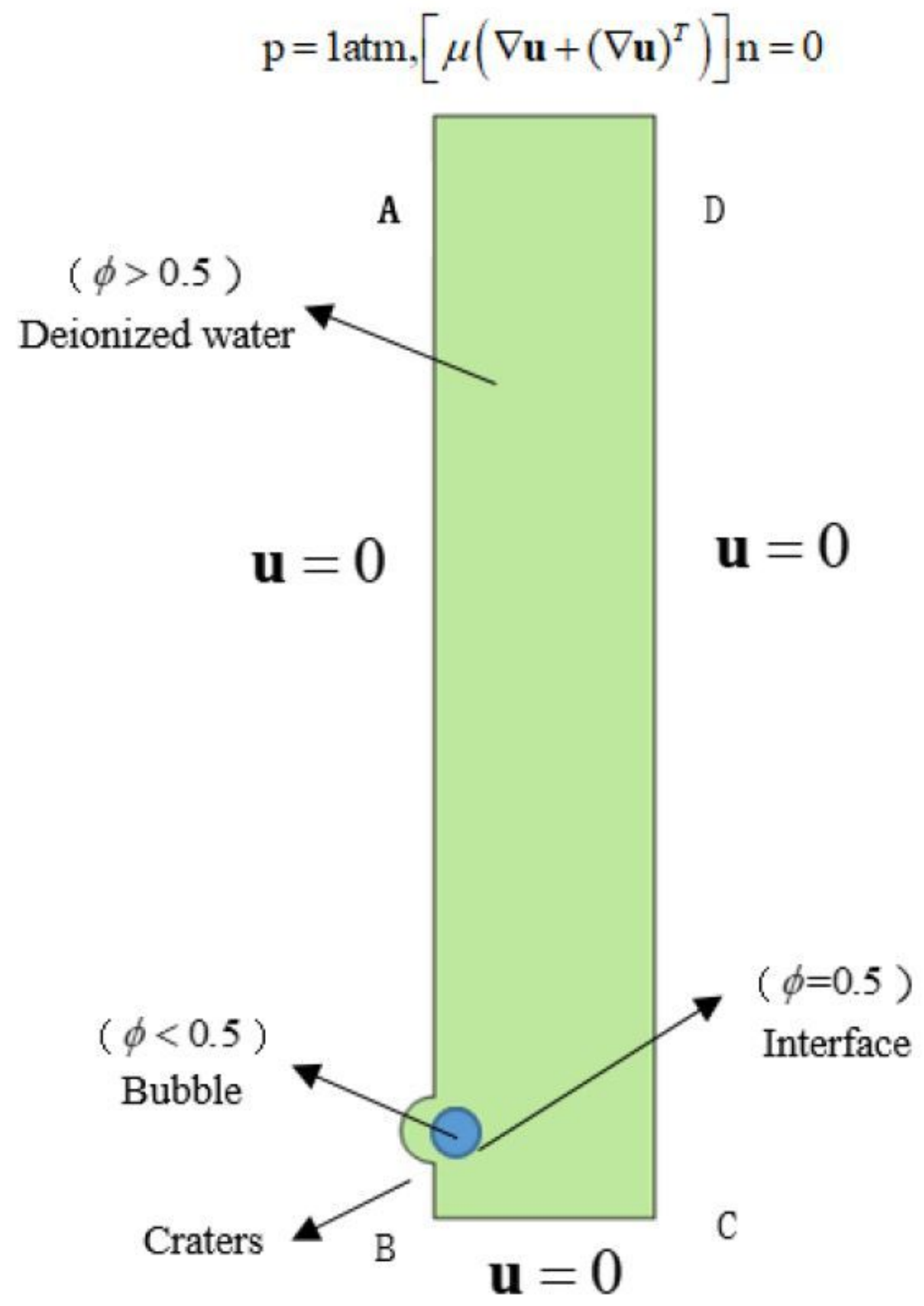

Figure 5

Schematic diagram of bubble tracking calculation 


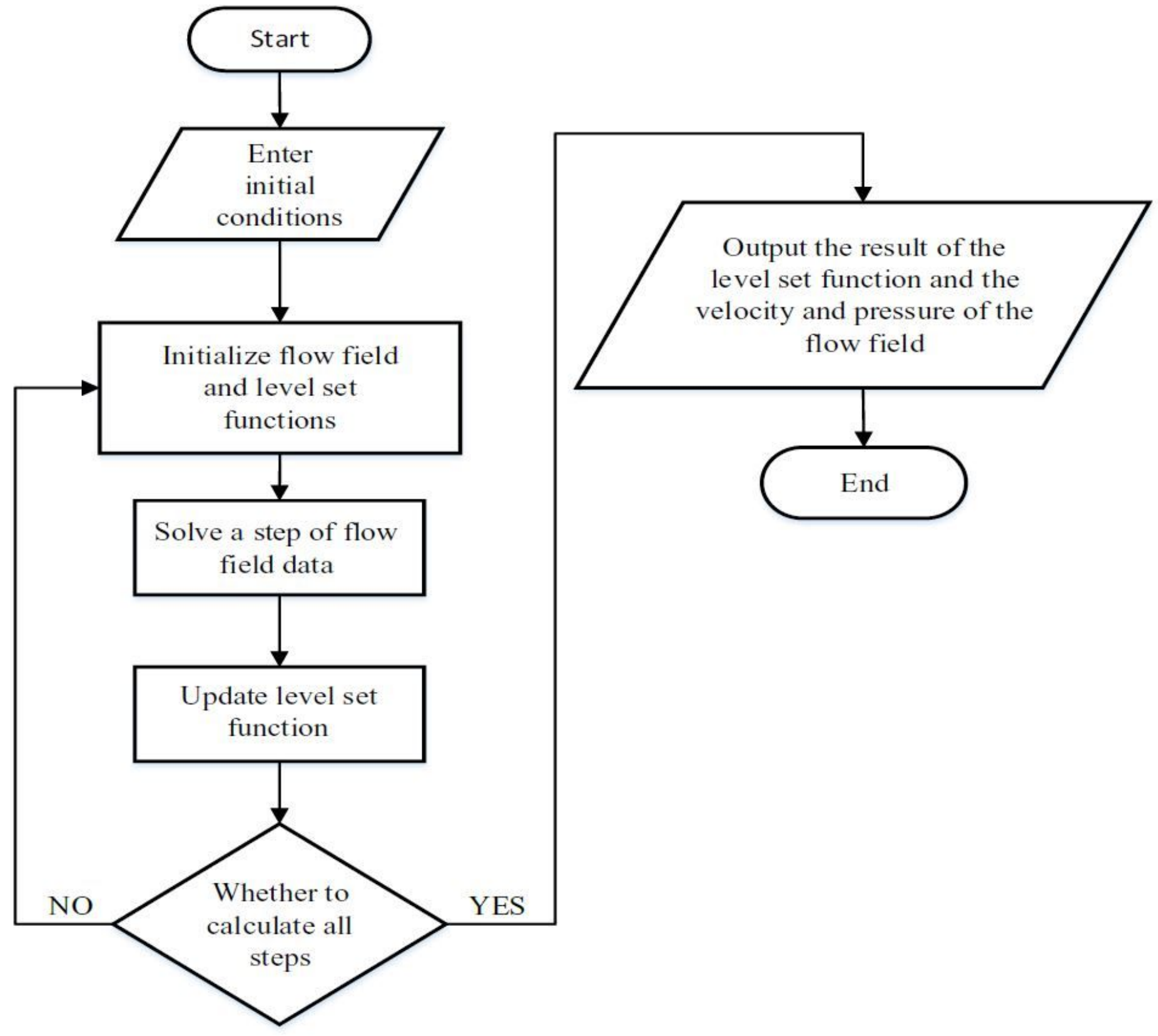

Figure 6

Flow chart of bubble tracking calculation 
Input :

- Servo voltage

- Peak current

- Magnetic field strength

- Ultrasonic vibration amplitude

- Fluid density

- Fluid viscosity

- Ultrasonic vibration amplitude

- Ultrasonic vibration frequency

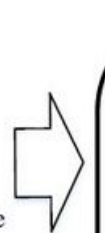

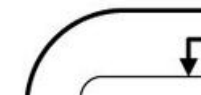

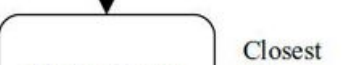

Electrode wire

vibration model

distance

Electric field

strength under

bubble

breakdown

mechanism

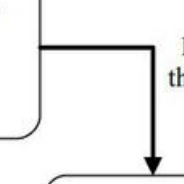

electric field

strength

Return

the result

initial

position

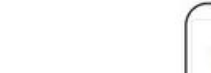

of

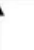

Return

the result
Output:

- discharge point position

Figure 7

Schematic of the calculation of discharge point 


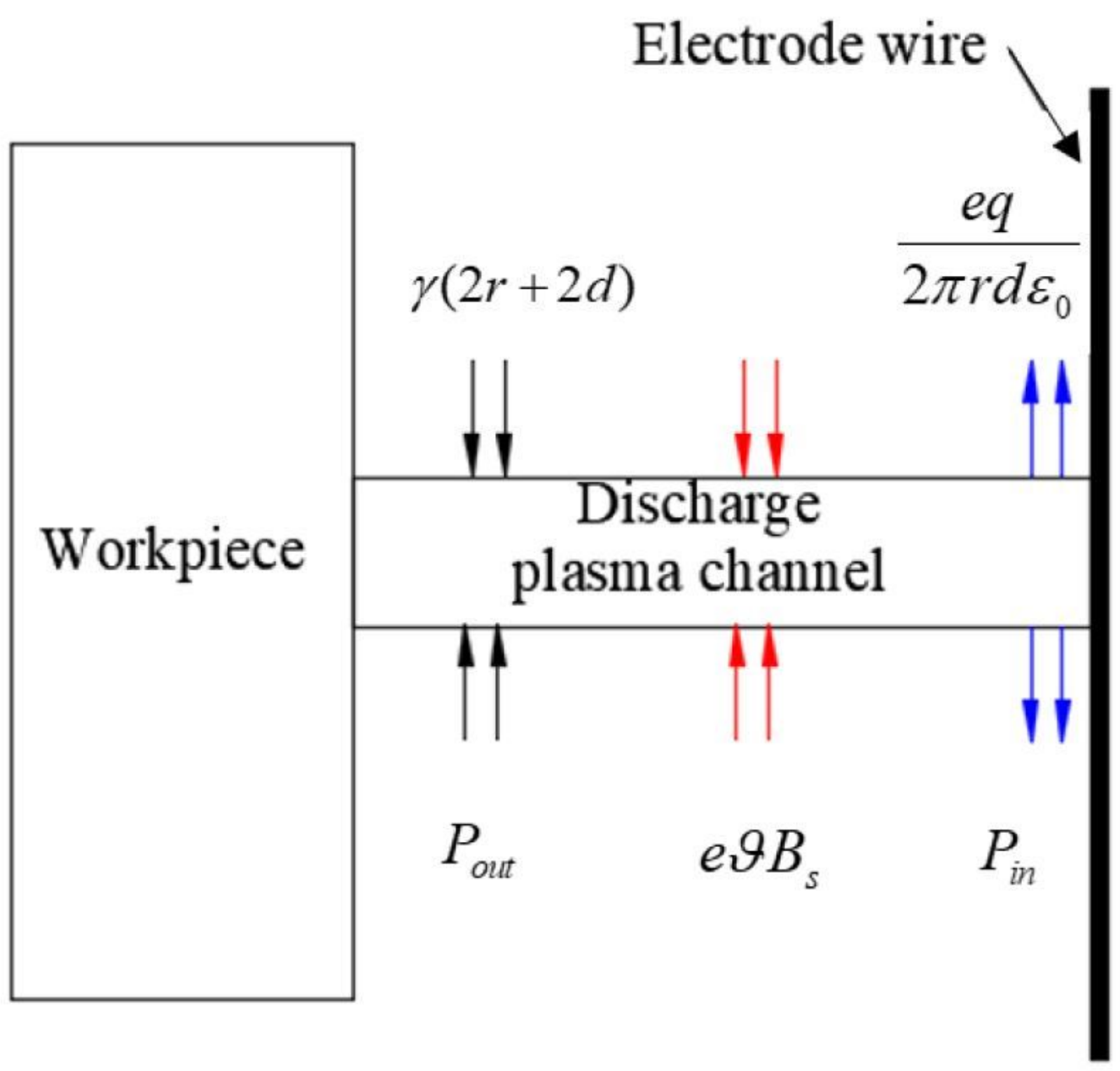

Figure 8

Schematic diagram of the discharge channel 


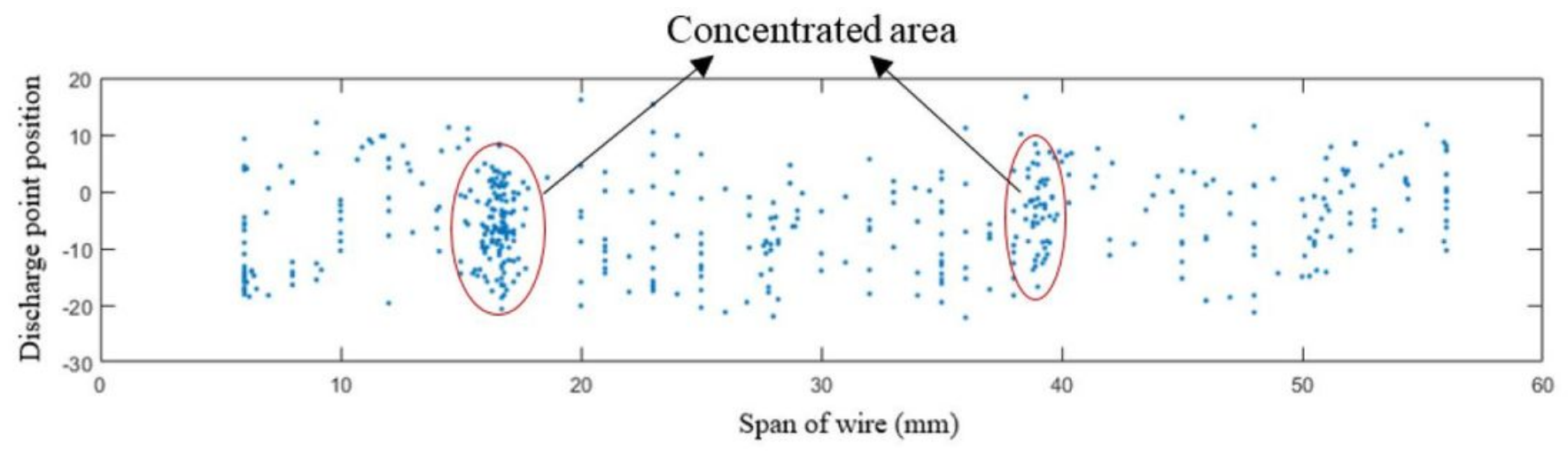

(a) Traditional WEDM-LS ( $\left.T_{\text {on }}=15 \mu \mathrm{s}, I P=6 \mathrm{~A}\right)$

(b)

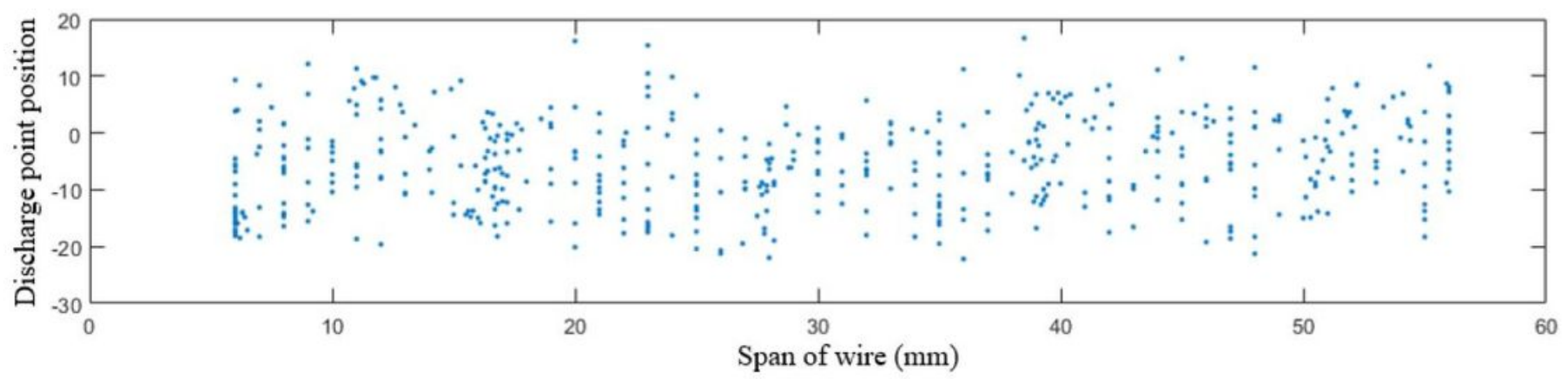

(c) USV-MF assisted WEDM-LS ( $\left.T_{\text {on }}=15 \mu \mathrm{s}, I P=6 \mathrm{~A}\right)$

Figure 9

The distribution of discharge position in discharge channel 


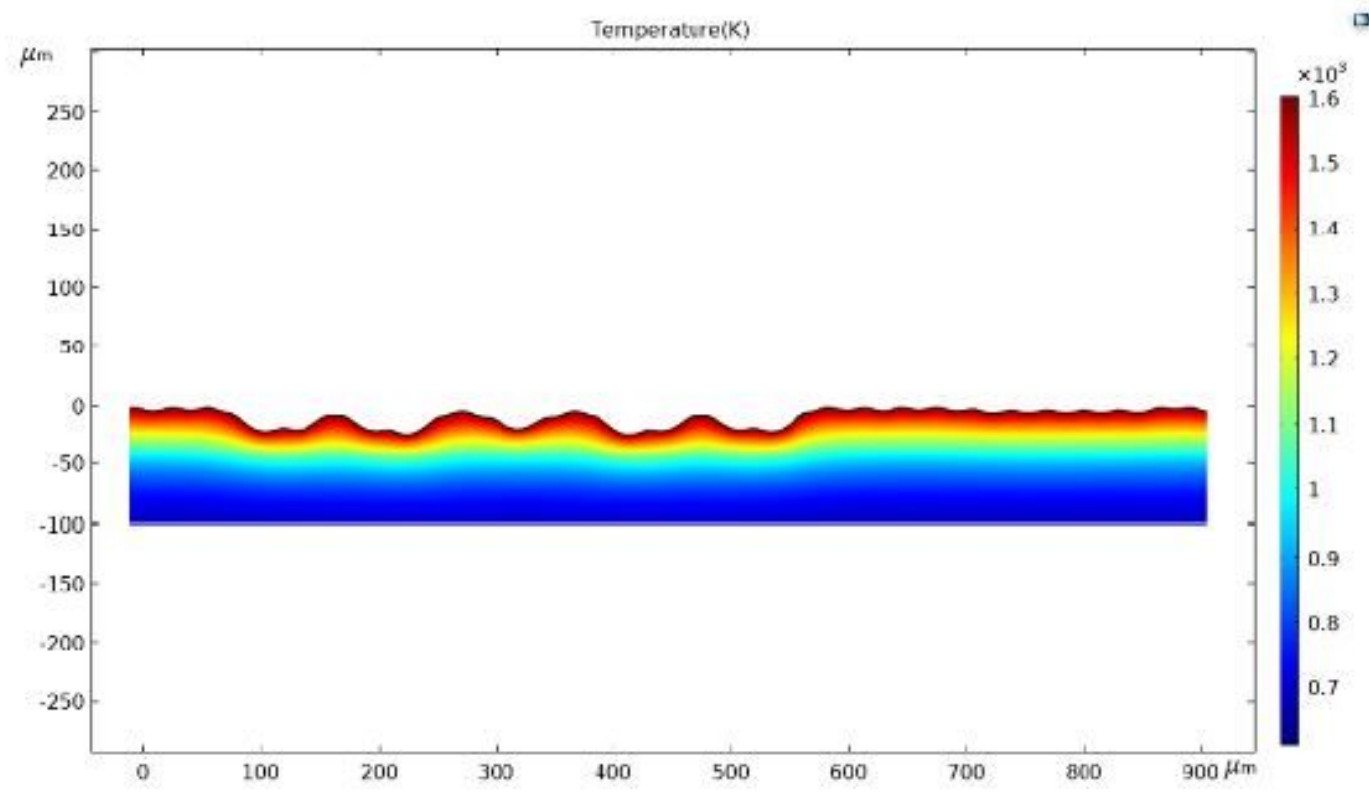

(a) Traditional WEDM-LS $\left(T_{\text {on }}=15 \mu \mathrm{s}, I P=10 \mathrm{~A}\right)$

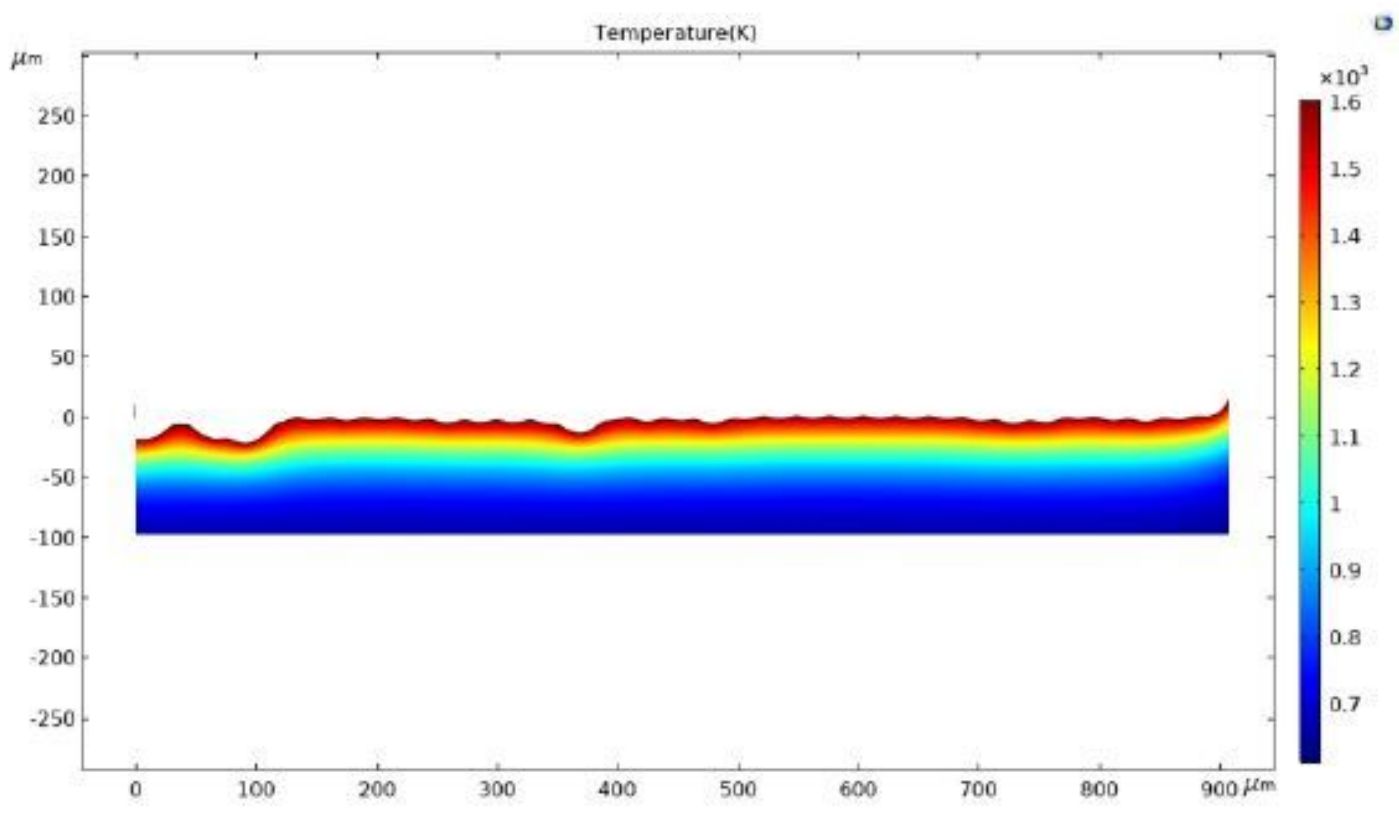

(b) USV-MF assisted WEDM-LS $\left(T_{\text {on }}=15 \mu \mathrm{s}, I P=10 \mathrm{~A}\right)$

Figure 10

Surface morphology of workpiece under continuous discharge machining 

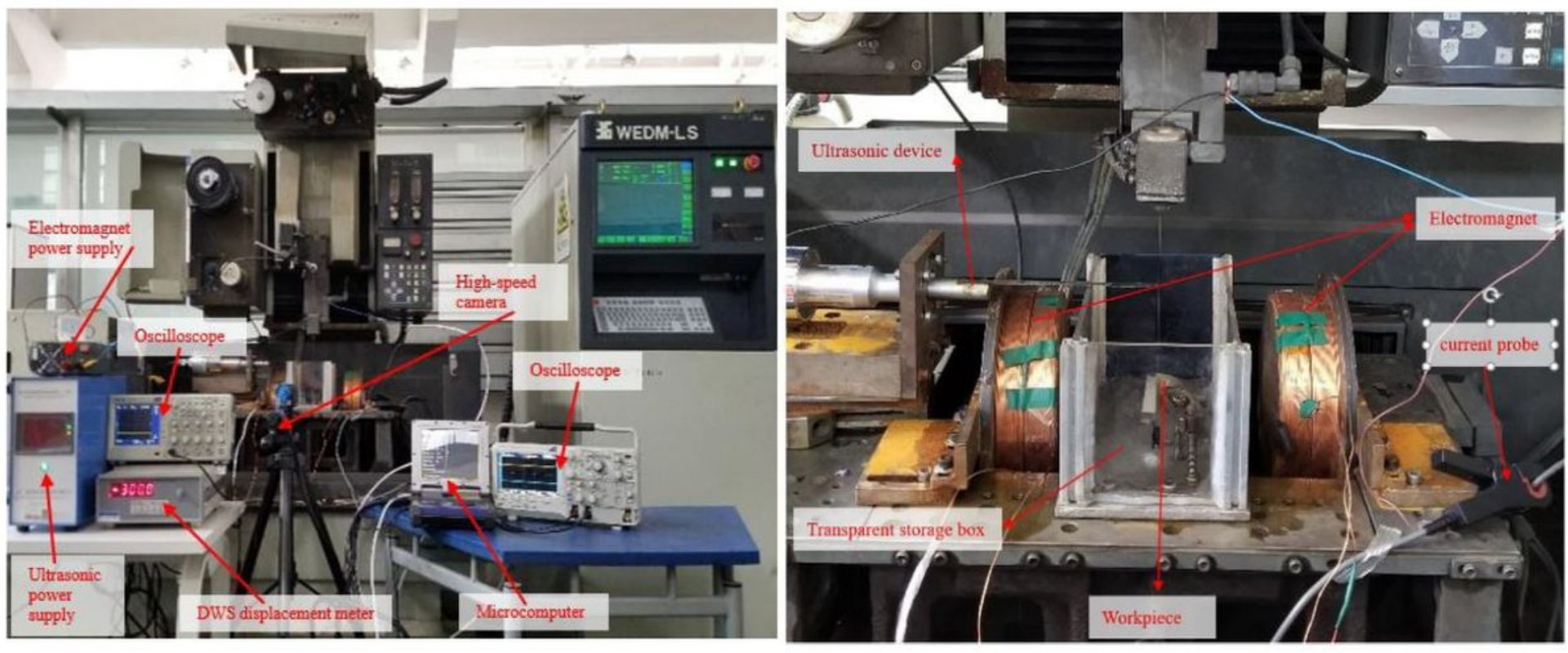

Figure 11

Experimental setup

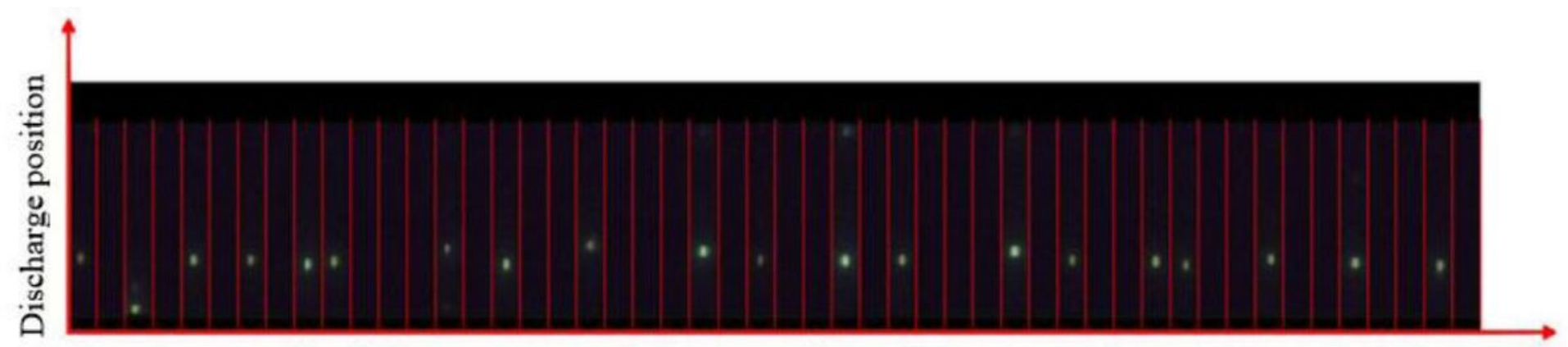

The serial number of the picture

(a) $0-50 \mathrm{fps}$

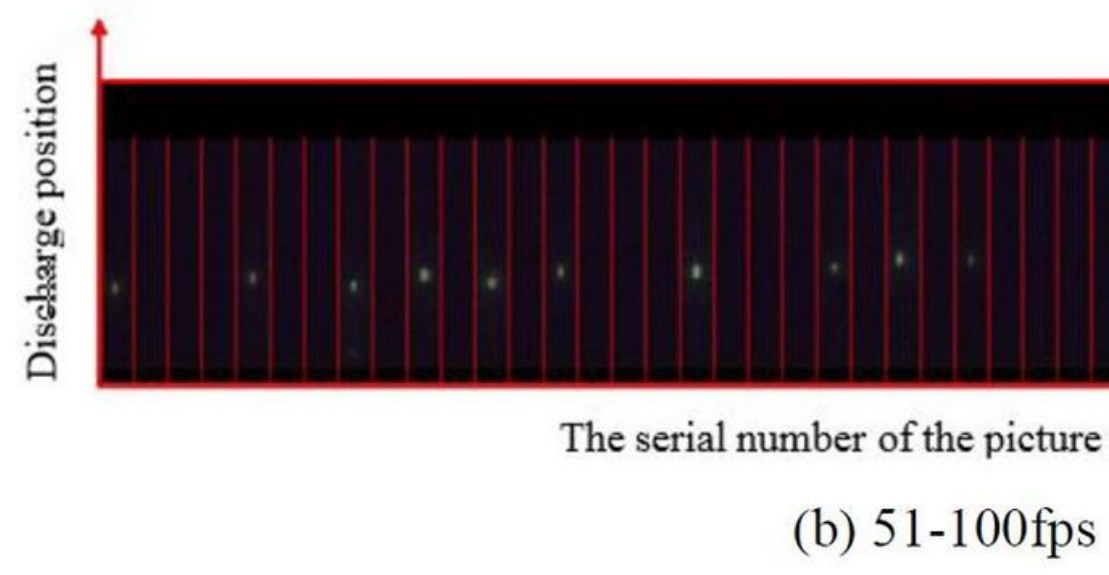

Figure 12

Distribution of discharge points in traditional WEDM-LS 


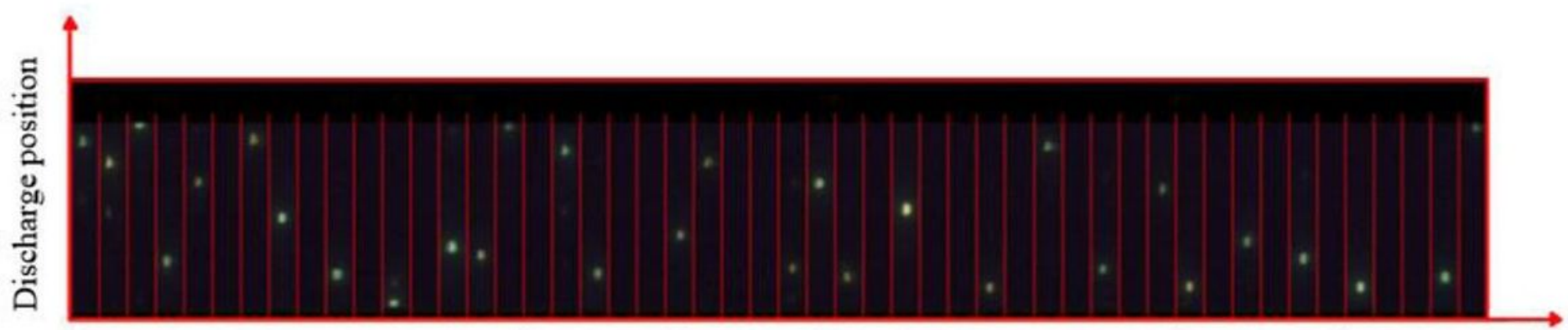

The serial number of the picture

(a) $0-50 \mathrm{fp}$

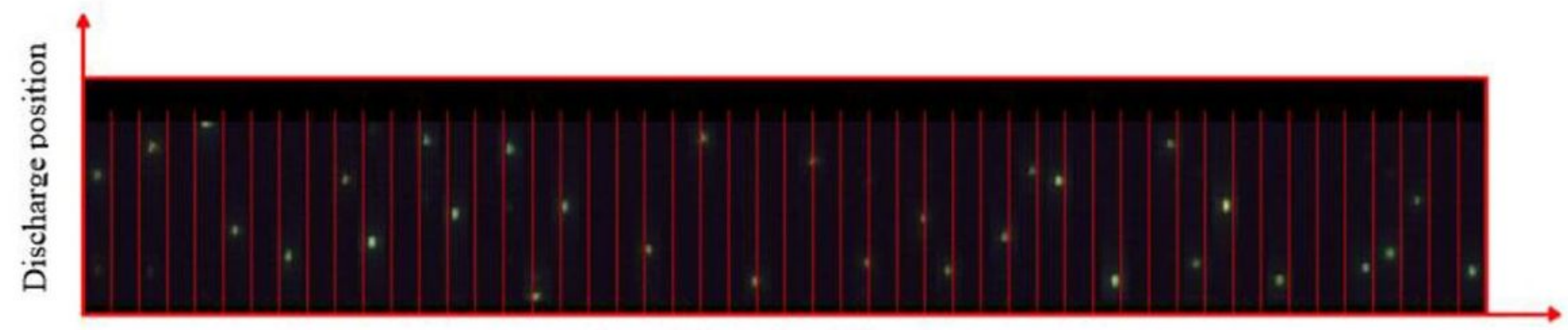

The serial number of the picture

(b) $51-100 \mathrm{fps}$

Figure 13

Distribution of discharge points in USV-MF assisted WEDM-LS 


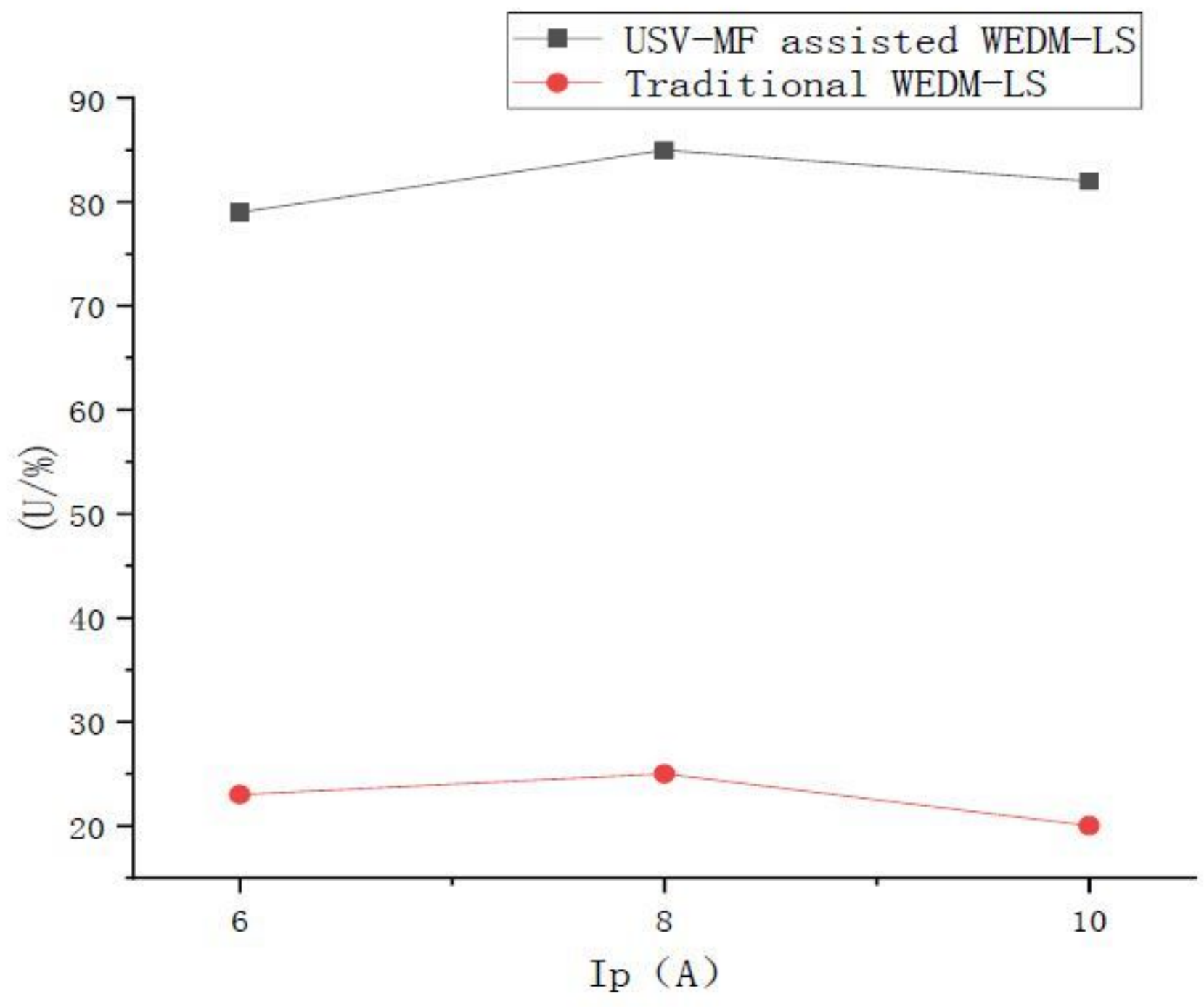

Figure 14

The uniformity coefficient under different machining parameters 
Abnormal discharge
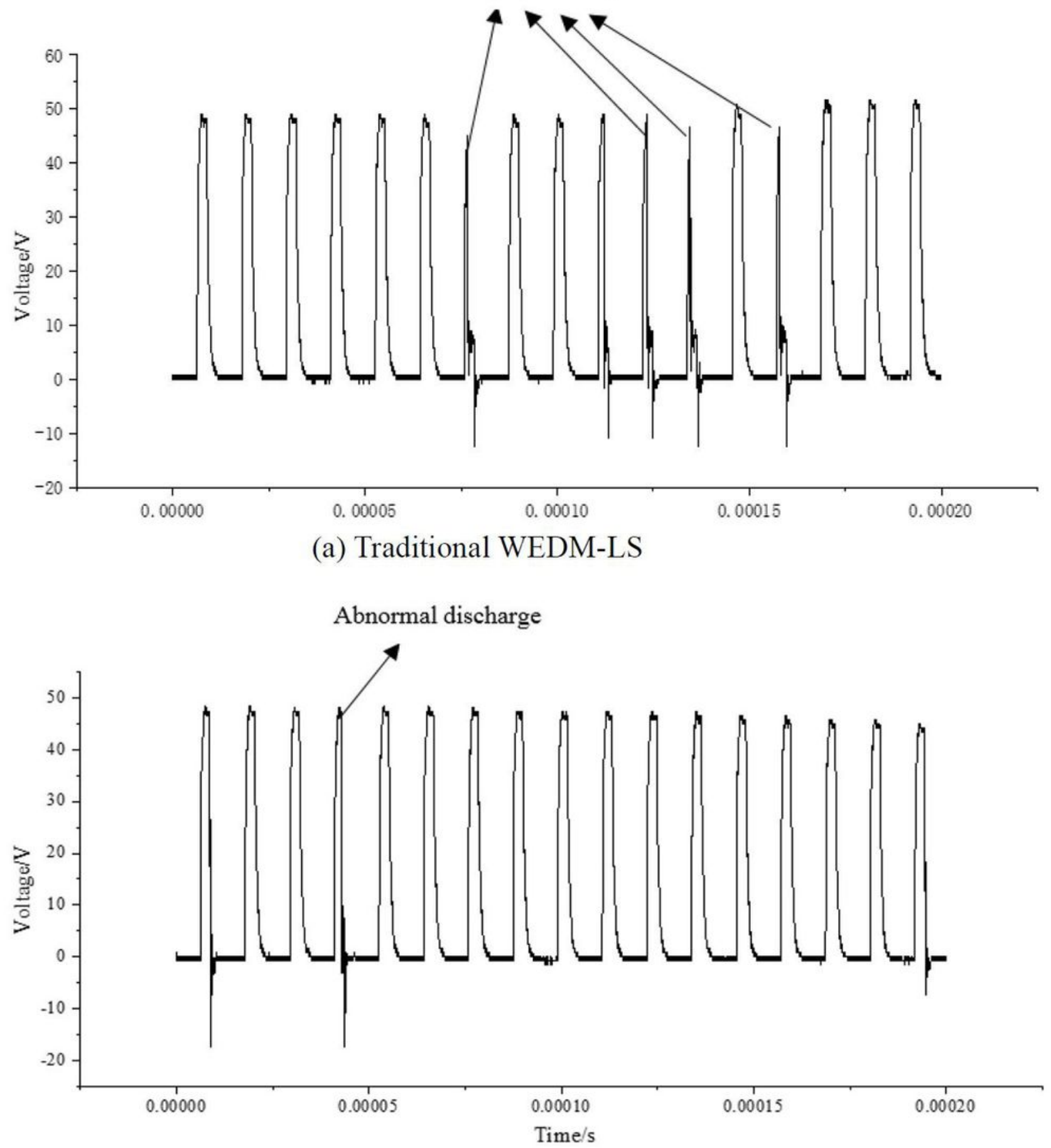

(d) USV-MF assisted WEDM-LS

Figure 15

Discharge waveform 


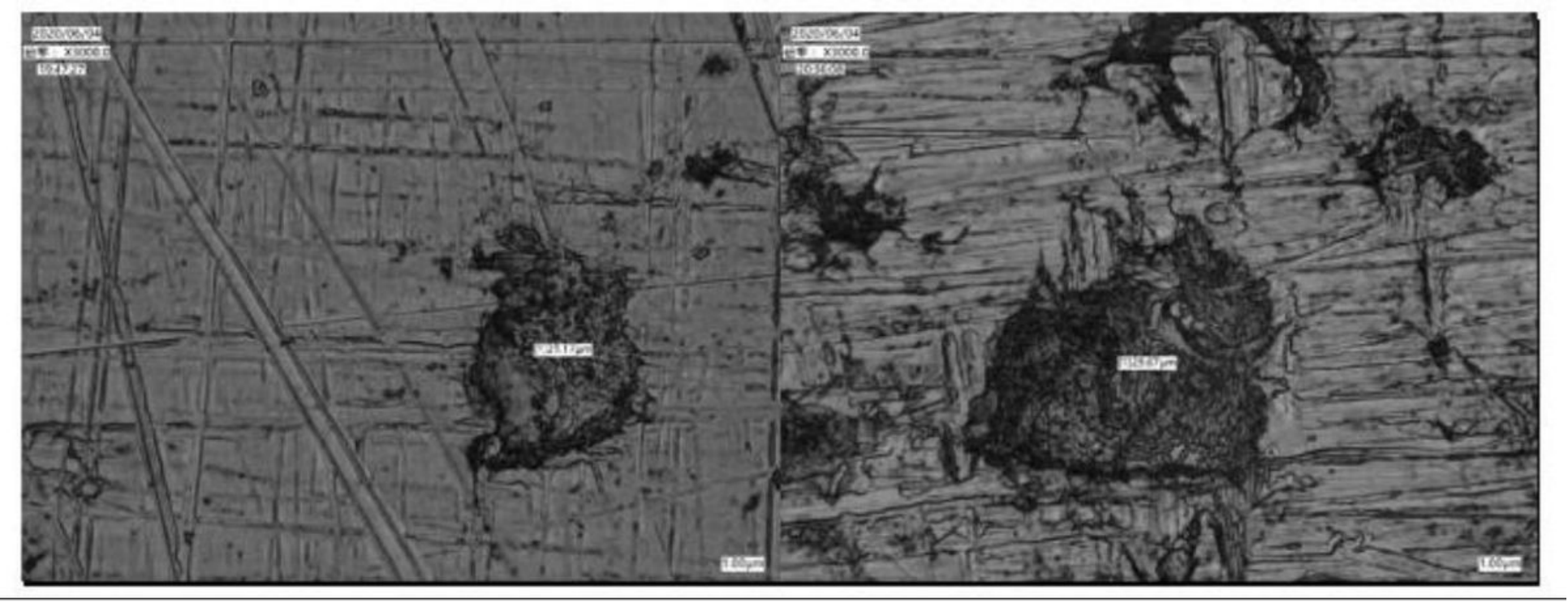

(a) Traditional WEDM-LS

(b) USV-MF assisted WEDM-LS

Figure 16

Crater diameter of workpiece $($ Ton $=15 \mu \mathrm{s}, \mathrm{IP}=10 \mathrm{~A})$ 


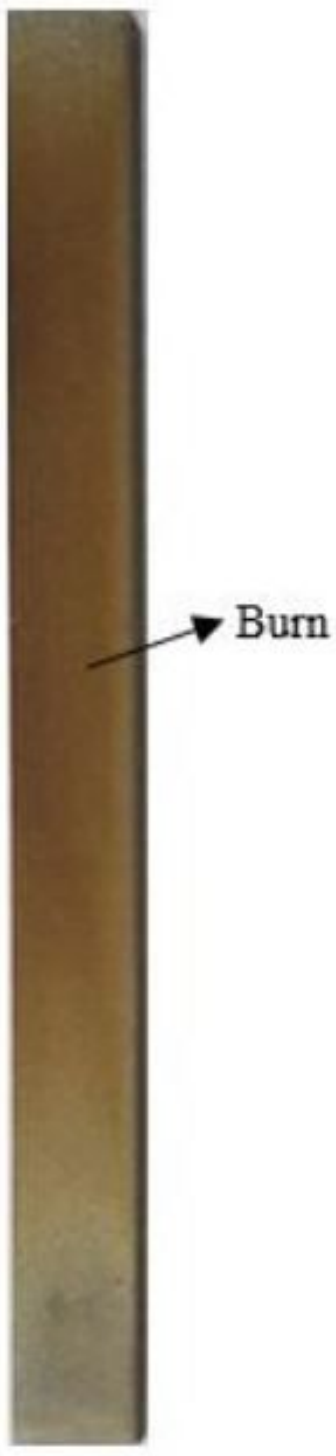

(a) Traditional WEDM-LS

(b) USV-MF assisted WEDM-LS

Figure 17

Workpiece surfaces machined in traditional WEDM-LS and USV-MF assisted WEDM-LS (Ton $=15 \mu \mathrm{s}$, $\mathrm{IP}=10 \mathrm{~A}$ ) 

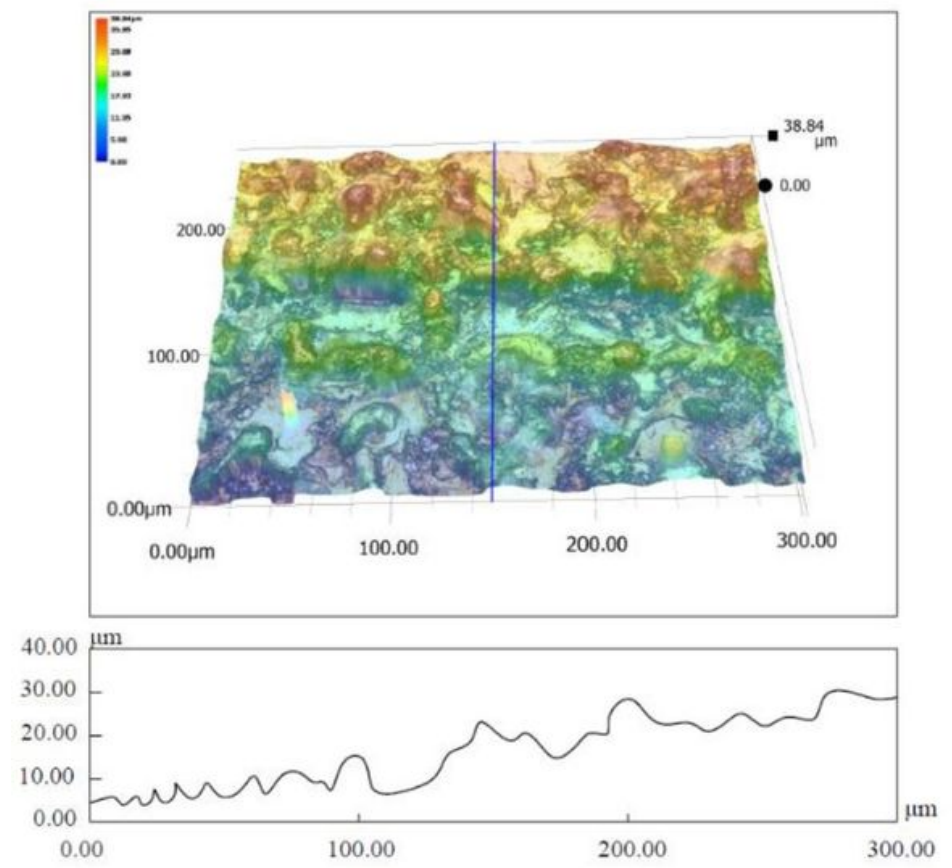

(a) Traditional WEDM-LS
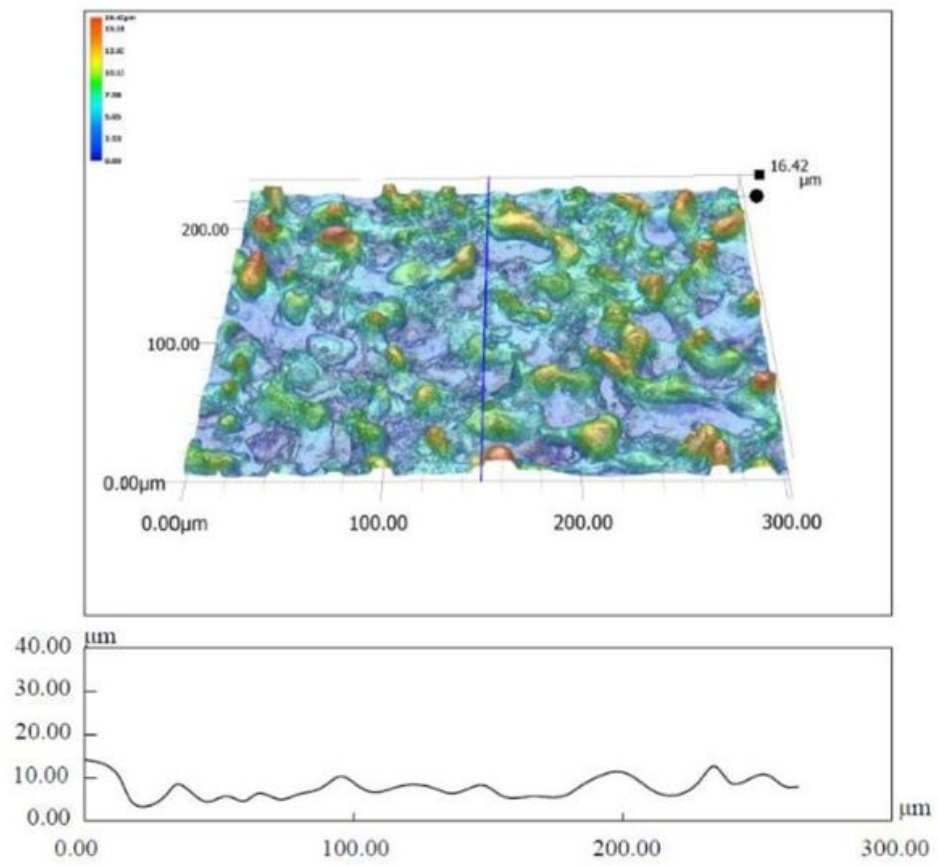

(b) USV-MF assisted WEDM-LS

Figure 18

$3 \mathrm{D}$ surface morphology of workpiece $($ Ton $=15 \mu \mathrm{s}, \mathrm{IP}=10 \mathrm{~A})$

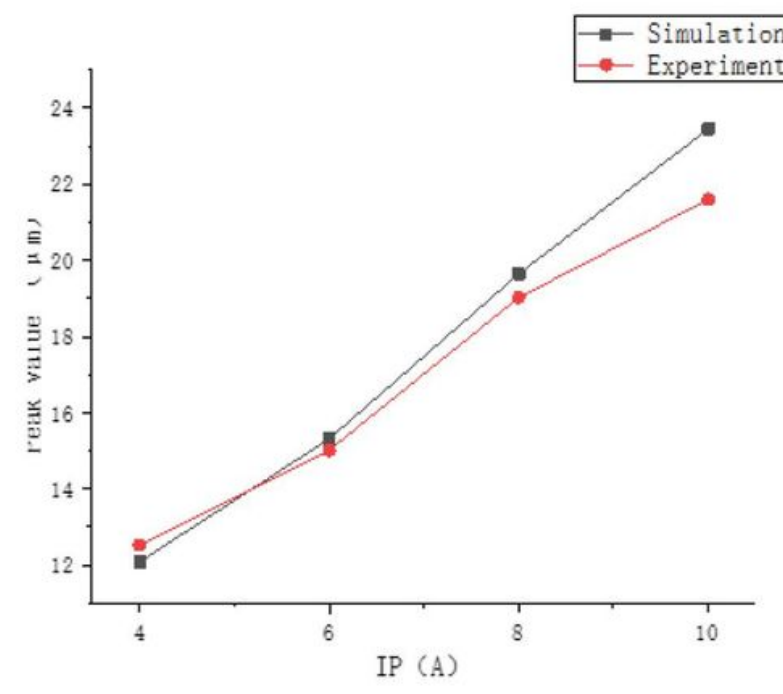

(a) Traditional WEDM-LS

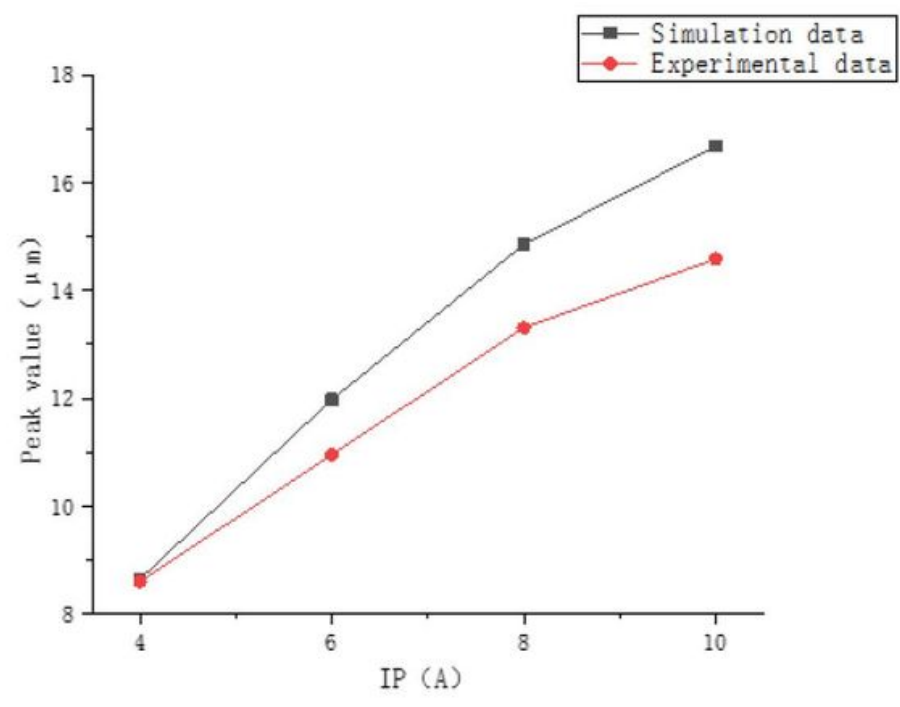

(b) USV-MF assisted WEDM-LS

Figure 19

Experimental and simulated surface peak values 